Proceedings of the Institute of Mathematics and Mechanics,

National Academy of Sciences of Azerbaijan

Volume 46, Number 2, 2020, Pages 243-271

https://doi.org/10.29228/proc.31

\title{
GLOBAL BOUNDEDNESS AND STABILITY OF SOLUTIONS OF NONAUTONOMOUS DEGENERATE DIFFERENTIAL EQUATIONS
}

\author{
MARIA S. FILIPKOVSKA (FILIPKOVSKAYA)
}

\begin{abstract}
For nonautonomous (time-varying) degenerate differential equations, which are also called nonautonomous differential-algebraic equations, conditions of the Lagrange stability and instability, the Lyapunov stability and instability, ultimate boundedness and asymptotic stability, including conditions of asymptotic stability in the large (or complete stability) are obtained. Note that the Lagrange stability of the equation (as well as the ultimate boundedness) guarantees its global solvability for all consistent initial values and the boundedness (the ultimate boundedness) of all its solutions. The Lagrange instability enable to identify solutions with a finite escape time, i.e., the solutions blowing up in finite time.
\end{abstract}

\section{Introduction}

In the present work, nonautonomous degenerate differential equations of the form

$$
\frac{d}{d t}[A(t) x(t)]+B(t) x(t)=f(t, x(t)),
$$

where $f:\left[t_{+}, \infty\right) \times \mathbb{R}^{n} \rightarrow \mathbb{R}^{n}, t_{+} \geq 0$, and $A, B:\left[t_{+}, \infty\right) \rightarrow L\left(\mathbb{R}^{n}\right)$ are studied. We do not require the operator $A(t)$ to be nondegenerate (invertible). Thus, in the general case, the operator $A(t)$ is degenerate and therefore the differential equation (DE) (1.1) is called degenerate. It is also called a semilinear (sometimes quasilinear) differential-algebraic equation (DAE). Equations of the type (1.1) with time-varying operator $A(t)$ are called time-varying or nonautonomous. Note that the operator $B(t)$ can also be degenerate.

The initial condition is given by

$$
x\left(t_{0}\right)=x_{0} \quad\left(t_{0} \geq t_{+}\right) .
$$

The initial point $\left(t_{0}, x_{0}\right)$ must satisfy a consistency condition which will be given below (see Remark 2.1).

A function $x \in C\left(\left[t_{0}, t_{1}\right), \mathbb{R}^{n}\right)$ is said to be a solution of the equation (1.1) on $\left[t_{0}, t_{1}\right)\left(\left[t_{0}, t_{1}\right) \subseteq\left[t_{+}, \infty\right)\right)$ if the function $A(t) x(t)$ is continuously differentiable on

2010 Mathematics Subject Classification. 34A09, 34D20, 34D23.

Key words and phrases. nonautonomous, global boundedness of solutions, Lagrange stability, ultimate boundedness, Lyapunov stability, asymptotic stability in the large, degenerate differential equation, differential-algebraic equation. 
$\left[t_{0}, t_{1}\right)$ and $x(t)$ satisfies $(1.1)$ on $\left[t_{0}, t_{1}\right)$. If the function $x(t)$ additionally satisfies the initial condition (1.2), then it is called a solution of the initial value problem (the Cauchy problem) (1.1), (1.2). If we consider the equation

$$
A(t) \frac{d}{d t} x(t)+B(t) x(t)=f(t, x(t))
$$

instead of (1.1), then its solution has to be smoother, namely, a function $x \in$ $C^{1}\left(\left[t_{0}, t_{1}\right), \mathbb{R}^{n}\right)$ satisfying (1.3) on $\left[t_{0}, t_{1}\right)$ is called a solution of the equation (1.3) on $\left[t_{0}, t_{1}\right)$. If $x(t)$ additionally satisfies (1.2), then it is called a solution of the initial value problem (1.3), (1.2).

Degenerate DEs or DAEs of type (1.1) describe various dynamical systems which are nonlinear and may have nonlinear algebraic (functional) relationships between the coordinates of variables and relationships between these variables and external influences. These equations are used to describe mathematical models in control theory (where they are called descriptor equations), radio electronics, robotics, economics, ecology and chemical kinetics (see, e.g., [26, 3, 2, 15, 19, 4, 14, 28]). Due to a wide range of applications, degenerate DEs (DAEs, algebraicdifferential systems, descriptor equations) have been studied by many authors. The local solvability was studied, e.g., in the monographs $[26,2,3,15,19,14]$ (also, see references therein), where various approaches to the study of such equations are presented. In $[3,15,19]$, the Lyapunov stability of the equilibrium position was also studied. The Lyapunov stability of the zero solution of an nonautonomous semilinear DAE under certain simplifications of the nonlinear function in the equation was considered in [25]. The global solvability of semilinear DAEs was studied in [11, 26, 3, 15].

The references cited, except for [26] and, in part, [15], concern the study of degenerate DEs in finite-dimensional spaces. It is worth noting that degenerate DEs of the type (1.1), (1.3) in infinite-dimensional spaces (operator-differential equations, implicit evolution equations, abstract DAEs) are important for the study of partial differential equations (PDEs), since any type of a PDE can be represented as a degenerate DE and, possibly, a complementary boundary condition (see, e.g., $[26,24,27])$. Degenerate parabolic and elliptic-parabolic equations were considered in [10,26, 24, 27], and in [26] degenerate hyperbolic equations were also considered.

The conditions of global solvability (including the uniqueness of global solutions) for autonomous degenerate DEs of the form (1.1) ( $A, B$ are time-invariant) were obtained by the author in [6] and, together with A.G. Rutkas, in [21] in the case of the regular characteristic pencil $\lambda A+B$, and in [9] in the case of the nonregular (singular) characteristic pencil. The conditions of Lagrange stability for autonomous degenerate DEs of the form (1.1) were obtained by the author in $[8,9]$. In [7], two combined numerical methods were presented and it was verified that the results of applying the theorems from [8] were consistent with the results of numerical experiments. In the present paper, the nonautonomous degenerate DEs (1.1) and (1.3) with characteristic pencils regular for every $t$ are studied.

The following notation is used in the paper: $I_{X}$ is the identity operator in $X$; $\operatorname{rk} A$ is the rank of the operator $A$; $\operatorname{Ker} A$ is the kernel (the null-space) of the operator $A ; \mathcal{R}(A)$ is the range (the image) of the operator $A ; \delta_{i j}$ is the Kronecker delta. 


\section{Preliminaries and basic definitions}

The definitions and facts presented in this section will be used hereinafter.

The spectral projectors of the type of the Riesz projectors. Let for each $t \geq t_{+}$the operator pencil $\lambda A(t)+B(t)$ be regular ( $\lambda$ is a complex parameter) and let there exist functions $C_{1}:\left[t_{+}, \infty\right) \rightarrow(0, \infty), C_{2}:\left[t_{+}, \infty\right) \rightarrow(0, \infty)$ such that for all $t \in\left[t_{+}, \infty\right)$ the pencil resolvent $R(\lambda, t)=(\lambda A(t)+B(t))^{-1}$ satisfies the constraint

$$
\|R(\lambda, t)\| \leq C_{1}(t), \quad|\lambda| \geq C_{2}(t) .
$$

The condition (2.1) means that either the point $\mu=0$ is a simple pole of the resolvent $(A(t)+\mu B(t))^{-1}$ (this is equivalent to the fact that $\lambda=\infty$ is a removable singular point of the resolvent $R(\lambda, t)$ ), or $\mu=0$ is a regular point of the pencil $A(t)+\mu B(t)$.

If the regular pencil satisfies (2.1), then for each $t \in\left[t_{+}, \infty\right)$ there exist the two pairs of mutually complementary projectors [22], [26, Subsection 3.3] $\left(P_{1}(t)+\right.$ $\left.P_{2}(t)=I_{\mathbb{R}^{n}}, P_{i}(t) P_{j}(t)=\delta_{i j} P_{i}(t) ; Q_{1}(t)+Q_{2}(t)=I_{\mathbb{R}^{n}}, Q_{i}(t) Q_{j}(t)=\delta_{i j} Q_{i}(t)\right)$

$$
\begin{array}{ll}
P_{1}(t)=\frac{1}{2 \pi i} \oint_{|\lambda|=C_{2}(t)} R(\lambda, t) d \lambda A(t), & P_{2}(t)=I_{\mathbb{R}^{n}}-P_{1}(t), \\
Q_{1}(t)=\frac{1}{2 \pi i} \oint_{|\lambda|=C_{2}(t)} A(t) R(\lambda, t) d \lambda, & Q_{2}(t)=I_{\mathbb{R}^{n}}-Q_{1}(t)
\end{array}
$$

which generate the direct decompositions of spaces

$$
\mathbb{R}^{n}=X_{1}(t) \dot{+} X_{2}(t), X_{j}(t)=P_{j}(t) \mathbb{R}^{n}, \mathbb{R}^{n}=Y_{1}(t) \dot{+} Y_{2}(t), Y_{j}(t)=Q_{j}(t) \mathbb{R}^{n}
$$

such that the pairs of subspaces $X_{1}(t), Y_{1}(t)$ and $X_{2}(t), Y_{2}(t)$ are invariant under $A(t), B(t)$ (i.e., $\left.A(t), B(t): X_{j}(t) \rightarrow Y_{j}(t), j=1,2\right)$. The restricted operators $A_{j}(t)=\left.A(t)\right|_{X_{j}(t)}: X_{j}(t) \rightarrow Y_{j}(t), B_{j}(t)=\left.B(t)\right|_{X_{j}(t)}: X_{j}(t) \rightarrow Y_{j}(t), j=1,2$, are such that $A_{2}(t)=0, A_{1}^{-1}(t)$ exists (if $X_{1}(t) \neq\{0\}$ ), and $B_{2}^{-1}(t)$ exists (if $\left.X_{2}(t) \neq\{0\}\right)$. The subspaces $X_{i}(t), Y_{j}(t)$ are such that $Y_{1}(t)=\mathcal{R}(A(t))(\mathcal{R}(A(t))$ is the range $A(t)), X_{2}(t)=\operatorname{Ker} A(t), Y_{2}(t)=B(t) X_{2}(t)$ and $X_{1}(t)=R(\lambda, t) Y_{1}(t)$, $|\lambda| \geq C_{2}(t)$. The projectors have the properties $A(t) P_{1}(t)=Q_{1}(t) A(t)=A(t)$, $A(t) P_{2}(t)=Q_{2}(t) A(t)=0, B(t) P_{j}(t)=Q_{j}(t) B(t), j=1,2$, and are real, since $A(t), B(t)$ are real.

Using the spectral projectors, for each $t \in\left[t_{+}, \infty\right)$ we obtain the auxiliary operator [22], [26, subsection 3.3]

$$
G(t)=A(t)+B(t) P_{2}(t)=A(t)+Q_{2}(t) B(t) \in L\left(\mathbb{R}^{n}\right)
$$

such that $G(t): X_{j}(t) \rightarrow Y_{j}(t) \quad\left(G(t) X_{j}(t)=Y_{j}(t)\right)$, there exists $G^{-1}(t)=A_{1}^{-1}(t) Q_{1}(t)+B_{2}^{-1}(t) Q_{2}(t) \in L\left(\mathbb{R}^{n}\right) \quad\left(G^{-1}(t): Y_{j}(t) \rightarrow X_{j}(t)\right)$, and $G^{-1}(t) A(t) P_{1}(t)=G^{-1}(t) A(t)=P_{1}(t), G^{-1}(t) B(t) P_{2}(t)=P_{2}(t)$, $A(t) G^{-1}(t) Q_{1}(t)=A(t) G^{-1}(t)=Q_{1}(t), B(t) G^{-1}(t) Q_{2}(t)=Q_{2}(t)$.

Note that the projection operator functions $P_{i}(t), Q_{i}(t)$ and the operator functions $G(t), G^{-1}(t)$ have the same degree of smoothness as the operator functions $A(t), B(t)$ and the function $C_{2}(t)$ [26, subsection 3.3]. Suppose that the operator functions $A(t), B(t)$ and the function $C_{2}(t)$ are continuously differentiable on $\left[t_{+}, \infty\right)$, i.e., $A, B \in C^{1}\left(\left[t_{+}, \infty\right), L\left(\mathbb{R}^{n}\right)\right)$ and $C_{2} \in C^{1}\left(\left[t_{+}, \infty\right),(0, \infty)\right)$, then the 
operator functions $P_{i}(t), Q_{i}(t), G(t)$ and $G^{-1}(t)$ are also continuously differentiable on $\left[t_{+}, \infty\right)$.

With respect to the decomposition (2.3), for each $t$ any vector $x \in \mathbb{R}^{n}$ can be uniquely represented in the form

$$
x=P_{1}(t) x+P_{2}(t) x=x_{p_{1}}(t)+x_{p_{2}}(t), \quad x_{p_{i}}(t)=P_{i}(t) x \in X_{i}(t) .
$$

Note that the equation (1.1) is equivalent to

$$
A(t)\left[P_{1}(t) x(t)\right]^{\prime}+A^{\prime}(t)\left[P_{1}(t) x(t)\right]+B(t) x(t)=f(t, x(t)) .
$$

Applying the projectors $Q_{1}(t), Q_{2}(t)$ to (1.1) and using their properties, we obtain the equivalent system

$$
\begin{aligned}
& A(t) P_{1}(t)\left[P_{1}(t) x(t)\right]^{\prime}+Q_{1}(t) A^{\prime}(t) P_{1}(t) x(t)+B(t) P_{1}(t) x(t)=Q_{1}(t) f(t, x(t)), \\
& Q_{2}(t) A^{\prime}(t) P_{1}(t) x(t)+B(t) P_{2}(t) x(t)=Q_{2}(t) f(t, x(t)) .
\end{aligned}
$$

Applying the operator $G^{-1}(t)$ to the system (2.6), (2.7) and using its properties, we obtain the equivalent system

$$
\begin{aligned}
& P_{1}(t)\left[P_{1}(t) x(t)\right]^{\prime}+G^{-1}(t) Q_{1}(t)\left[A^{\prime}(t)+B(t)\right] P_{1}(t) x(t)=G^{-1}(t) Q_{1}(t) f(t, x(t)), \\
& P_{2}(t) x(t)=G^{-1}(t) Q_{2}(t)\left[f(t, x(t))-A^{\prime}(t) P_{1}(t) x(t)\right] .
\end{aligned}
$$

Taking into account the equality $P_{1}(t)\left[P_{1}(t) x(t)\right]^{\prime}=\left[P_{1}(t) x(t)\right]^{\prime}-P_{1}^{\prime}(t) P_{1}(t) x(t)$, the obtained system can be rewritten as

$$
\begin{aligned}
& {\left[P_{1}(t) x(t)\right]^{\prime}=\left[P_{1}^{\prime}(t)-G^{-1}(t) Q_{1}(t)\left[A^{\prime}(t)+B(t)\right]\right] P_{1}(t) x(t)+} \\
& +G^{-1}(t) Q_{1}(t) f(t, x(t)), \\
& G^{-1}(t) Q_{2}(t)\left[f(t, x(t))-A^{\prime}(t) P_{1}(t) x(t)\right]-P_{2}(t) x(t)=0 .
\end{aligned}
$$

Thus, the degenerate DE (1.1) is reduced to the equivalent system (2.8). Using the representation (2.5), we can write the system (2.8) in the form

$$
\begin{aligned}
& x_{p_{1}}^{\prime}(t)=\left[P_{1}^{\prime}(t)-G^{-1}(t) Q_{1}(t)\left[A^{\prime}(t)+B(t)\right]\right] x_{p_{1}}(t)+ \\
& +G^{-1}(t) Q_{1}(t) f\left(t, x_{p_{1}}(t)+x_{p_{2}}(t)\right), \\
& G^{-1}(t) Q_{2}(t)\left[f\left(t, x_{p_{1}}(t)+x_{p_{2}}(t)\right)-A^{\prime}(t) x_{p_{1}}(t)\right]-x_{p_{2}}(t)=0 .
\end{aligned}
$$

The equation (1.3) is transformed analogously. Applying the projectors $Q_{1}(t)$, $Q_{2}(t)$ to (1.3), we obtain the equivalent system

$$
\begin{aligned}
& A(t) P_{1}(t) x^{\prime}(t)+B(t) P_{1}(t) x(t)=Q_{1}(t) f(t, x(t)), \\
& B(t) P_{2}(t) x(t)=Q_{2}(t) f(t, x(t)) .
\end{aligned}
$$

Further, using $G^{-1}(t)$, we transform the obtained system to the equivalent system

$$
\begin{aligned}
& {\left[P_{1}(t) x(t)\right]^{\prime}=G^{-1}(t)\left[-B(t) P_{1}(t) x(t)+Q_{1}(t) f(t, x(t))\right]+P_{1}^{\prime}(t) x(t),} \\
& G^{-1}(t) Q_{2}(t) f(t, x(t))-P_{2}(t) x(t)=0 .
\end{aligned}
$$

Thus, the degenerate DE (1.3) is reduced to the equivalent system (2.13). Using the representation $(2.5)$, we can write $(2.13)$ in the form

$$
\begin{aligned}
& x_{p_{1}}^{\prime}(t)=G^{-1}(t)\left[-B(t) x_{p_{1}}(t)+Q_{1}(t) f\left(t, x_{p_{1}}(t)+x_{p_{2}}(t)\right)\right]+ \\
& +P_{1}^{\prime}(t)\left(x_{p_{1}}(t)+x_{p_{2}}(t)\right), \\
& G^{-1}(t) Q_{2}(t) f\left(t, x_{p_{1}}(t)+x_{p_{2}}(t)\right)-x_{p_{2}}(t)=0 .
\end{aligned}
$$


From the foregoing, the remark follows:

Remark 2.1. Introduce the manifolds (the varieties)

$$
\begin{gathered}
L_{t_{+}}=\left\{(t, x) \in\left[t_{+}, \infty\right) \times \mathbb{R}^{n} \mid Q_{2}(t)\left[B(t) x+A^{\prime}(t) P_{1}(t) x-f(t, x)\right]=0\right\}, \\
\widehat{L}_{t_{+}}=\left\{(t, x) \in\left[t_{+}, \infty\right) \times \mathbb{R}^{n} \mid Q_{2}(t)[B(t) x-f(t, x)]=0\right\} .
\end{gathered}
$$

The consistency condition $\left(t_{0}, x_{0}\right) \in L_{t_{+}}\left(\left(t_{0}, x_{0}\right) \in \widehat{L}_{t_{+}}\right)$for the initial point $\left(t_{0}, x_{0}\right)$ is one of the necessary conditions for the existence of a solution of the initial value problem (1.1), (1.2) (the initial value problem (1.3), (1.2)). The initial point $\left(t_{0}, x_{0}\right)$ satisfying this condition is called consistent (the corresponding initial values $t_{0}, x_{0}$ are also called consistent).

The La Salle method for the continuation of solutions by using functions of the Lyapunov type. First we give some classic definitions from [17]. Let $D \subset$ $\mathbb{R}^{n}$ be a region containing the origin. A function $W \in C(D, \mathbb{R})$ is called positive definite if $W(0)=0$ and $W(x)>0$ for all $x \neq 0$. A function $V \in C\left(\left[t_{+}, \infty\right) \times D, \mathbb{R}\right)$ is called positive definite if $V(t, 0) \equiv 0$ and there exists a positive definite scalar function $W(x)$ such that $V(t, x) \geq W(x)$ for all $x \neq 0, t \in\left[t_{+}, \infty\right)$. Suppose that $V(t, x)$ has continuous first-order partial derivatives. The derivative of the function $V$ along the trajectories of the system (or the derivative of $V$ with respect to the system)

$$
x^{\prime}=f(t, x)
$$

is

$$
V_{(2.18)}^{\prime}(t, x)=\frac{\partial V}{\partial t}(t, x)+\left(\frac{\partial V}{\partial x}(t, x), f(t, x)\right) .
$$

By extending the second method of Lyapunov, in [16] J. La Salle obtained the theorems on the global solvability (solutions defined in the future) and also the Lagrange stability and instability of the ordinary differential equation (ODE) (2.18) $\left(f:[0, \infty) \times \mathbb{R}^{n} \rightarrow \mathbb{R}^{n}\right)$, where differential inequalities of the form

$$
v^{\prime} \leq \chi(t, v), \quad t \geq 0, \quad \text { and } \quad v^{\prime} \geq \chi(t, v), \quad t \geq 0,
$$

$\chi \in C([0, \infty) \times(0, \infty), \mathbb{R})$, were used. To apply these results, it is usually convenient to take $\chi(t, v)=k(t) U(v)$, where $k \in C([0, \infty), \mathbb{R})$ and $U \in C(0, \infty)$ $(U \in C((0, \infty), \mathbb{R})$ is a positive function). Then the inequalities (2.19) take the form

$$
\begin{aligned}
& \frac{v^{\prime}}{U(v)} \leq k(t), \quad t \geq 0, \\
& \frac{v^{\prime}}{U(v)} \geq k(t), \quad t \geq 0,
\end{aligned}
$$

and the following statements are true [16]: if $\int_{c}^{\infty} \frac{d v}{U(v)}=\infty(c=$ const $>0)$, then the inequality $(2.20)$ has no positive solution $v(t)$ with finite escape time; if $\int_{c}^{\infty} \frac{d v}{U(v)}=\infty$ and $\int_{t_{0}}^{\infty} k(t) d t<\infty\left(t_{0} \geq 0\right)$, then (2.20) has no unbounded positive solution for $t \geq 0$; if $\int_{c}^{\infty} \frac{d v}{U(v)}<\infty$ and $\int_{t_{0}}^{\infty} k(t) d t=\infty$, then (2.21) has no positive solution defined in the future (i.e., global). 


\section{Basic definitions.}

In [16], the definitions of a solution defined in the future, a solution with finite escape time and the Lagrange stability of the explicit ODE were given. Let us introduce similar definitions for degenerate DEs (DAEs).

A solution $x(t)$ of the initial value problem (1.1), (1.2) is called global or defined in the future if it exists on the interval $\left[t_{0}, \infty\right)$. A solution $x(t)$ of $(1.1),(1.2)$ is called Lagrange stable if it is global and bounded, i.e., $x(t)$ exists on $\left[t_{0}, \infty\right)$ and $\sup \|x(t)\|<\infty[8]$. $t \in\left[t_{0}, \infty\right)$

A solution $x(t)$ of the initial value problem (1.1), (1.2) has a finite escape time (the solution is blow-up in finite time) if it exists on some finite interval $\left[t_{0}, T\right)$ and is unbounded, i.e., there exists $T>t_{0}(T<\infty)$ such that $\lim _{t \rightarrow T-0}\|x(t)\|=\infty$. A solution $x(t)$ of $(1.1),(1.2)$ is called Lagrange unstable if it has a finite escape time [8].

The equation (1.1) is Lagrange stable for the initial point $\left(t_{0}, x_{0}\right)$ if for this initial point the solution of the initial value problem (1.1), (1.2) is Lagrange stable.

The equation (1.1) is called Lagrange stable if every solution of the initial value problem (1.1), (1.2) is Lagrange stable (i.e., the equation is Lagrange stable for every consistent initial point) [8].

The equation (1.1) is Lagrange unstable for the initial point $\left(t_{0}, x_{0}\right)$ if for this initial point the solution of the initial value problem (1.1), (1.2) is Lagrange unstable.

The equation (1.1) is called Lagrange unstable if every solution of the initial value problem (1.1), (1.2) is Lagrange unstable [8].

Similar definitions hold for the equation (1.3) (the initial value problem (1.3), $(1.2))$.

Definition 2.1. Consider an operator function $H:\left[t_{+}, \infty\right) \rightarrow L(X)$, where $X$ is a finite-dimensional linear space or Hilbert space. Let the operator $H(t) \in L(X)$ be self-adjoint for every $t \in\left[t_{+}, \infty\right)$. By analogy with [5], we introduce the following definitions. The operator $H(t)$ is called positive if $(H(t) x, x)>0$ for all $x \neq 0$, $t \in\left[t_{+}, \infty\right)$. The operator $H(t)$ is called uniformly positive or positive definite if there exists a constant $H_{0}>0$ such that $(H(t) x, x) \geq H_{0}\|x\|^{2}$ for all $x, t$.

If $X$ is a finite-dimensional linear space and a self-adjoint operator $H \in L(X)$ is time-invariant and positive (i.e., $(H x, x)>0$ for all $x \neq 0$ ), then it is also positive definite (uniformly positive). Clearly, $(H x, x) \geq H_{0}\|x\|^{2}$, where $H_{0}=\inf _{\|x\|=1}(H x, x)>0[5]$. For the time-varying operator $H(t)$ we can take $H_{0}=\inf _{\|x\|=1, t \in\left[t_{+}, \infty\right)}(H(t) x, x)$.

Remark 2.2. If we take the time-invariant self-adjoint operator $H \in L\left(\mathbb{R}^{n}\right)$ in the theorems presented in the paper, then it suffices to require that it be positive. 


\section{Global boundedness of solutions of nonautonomous degenerate differential equations}

In subsection 3.1, the conditions for the unique global solvability of the equation (1.1) are given. In subsection 3.2, the conditions for the Lagrange stability, which include the conditions of the unique global solvability and contain the conditions for the boundedness of global solutions (for all consistent initial points), are presented, as well as the conditions for the Lagrange instability under which the equation (1.1) has no global solutions (for consistent initial points $\left(t_{0}, x_{0}\right)$, where $P_{1}\left(t_{0}\right) x_{0}$ from a certain region $\Omega$ ) are presented. In subsection 3.3 , the conditions for the ultimate boundedness of solutions (dissipativity) of (1.1) are given. The corresponding results for the equation (1.3) are given in subsection 3.4.

\subsection{Global solvability.}

Theorem 3.1. Let $f \in C\left(\left[t_{+}, \infty\right) \times \mathbb{R}^{n}, \mathbb{R}^{n}\right), \frac{\partial}{\partial x} f \in C\left(\left[t_{+}, \infty\right) \times \mathbb{R}^{n}, L\left(\mathbb{R}^{n}\right)\right)$, $A, B \in C^{1}\left(\left[t_{+}, \infty\right), L\left(\mathbb{R}^{n}\right)\right)$, the pencil $\lambda A(t)+B(t)$ satisfy (2.1), where $C_{2} \in C^{1}\left(\left[t_{+}, \infty\right),(0, \infty)\right)$, and the following conditions be satisfied:

1) for each $t \in\left[t_{+}, \infty\right)$ and each $x_{p_{1}}(t) \in X_{1}(t)$ there exists a unique $x_{p_{2}}(t) \in X_{2}(t)$ such that

$$
\left(t, x_{p_{1}}(t)+x_{p_{2}}(t)\right) \in L_{t_{+}}
$$

2) for any fixed $t_{*} \in\left[t_{+}, \infty\right), x_{p_{1}}^{*}\left(t_{*}\right) \in X_{1}\left(t_{*}\right), x_{p_{2}}^{*}\left(t_{*}\right) \in X_{2}\left(t_{*}\right)$ such that $\left(t_{*}, x_{p_{1}}^{*}\left(t_{*}\right)+x_{p_{2}}^{*}\left(t_{*}\right)\right) \in L_{t_{+}}$the operator

$$
\Phi_{t_{*}, x_{p_{1}}^{*}\left(t_{*}\right), x_{p_{2}}^{*}\left(t_{*}\right)}=\left[\frac{\partial}{\partial x}\left[Q_{2}\left(t_{*}\right) f\left(t_{*}, x_{p_{1}}^{*}\left(t_{*}\right)+x_{p_{2}}^{*}\left(t_{*}\right)\right)\right]-B\left(t_{*}\right)\right] P_{2}\left(t_{*}\right)
$$

acting from $X_{2}\left(t_{*}\right)$ into $Y_{2}\left(t_{*}\right)$ has the inverse $\Phi_{t_{*}, x_{p_{1}}^{*}\left(t_{*}\right), x_{p_{2}}^{*}\left(t_{*}\right)}^{-1} \in$ $L\left(Y_{2}\left(t_{*}\right), X_{2}\left(t_{*}\right)\right)$;

3) there exist a positive definite self-adjoint operator $H(t) \in L\left(\mathbb{R}^{n}\right)$, a number $R>0$ and functions $U \in C(0, \infty), \quad k \in C\left(\left[t_{+}, \infty\right), \mathbb{R}\right)$ such that $H \in C^{1}\left(\left[t_{+}, \infty\right), L\left(\mathbb{R}^{n}\right)\right), \quad \int_{c}^{\infty} \frac{d v}{U(v)}=\infty \quad(c=$ const $>0)$ and the inequality $J\left(t, P_{1}(t) x\right) \leq k(t) U\left(\left(H(t) P_{1}(t) x, P_{1}(t) x\right)\right)$, where

$$
\begin{aligned}
J\left(t, P_{1}(t) x\right)= & \frac{1}{2}\left(H^{\prime}(t) P_{1}(t) x, P_{1}(t) x\right)+\left(H(t) P_{1}(t) x,\left[P_{1}^{\prime}(t)-\right.\right. \\
& \left.\left.-G^{-1}(t) Q_{1}(t)\left[A^{\prime}(t)+B(t)\right]\right] P_{1}(t) x+G^{-1}(t) Q_{1}(t) f(t, x)\right),
\end{aligned}
$$

holds for all $(t, x) \in L_{t_{+}}$such that $\left\|P_{1}(t) x\right\| \geq R$.

Then for each initial point $\left(t_{0}, x_{0}\right) \in L_{t_{+}}$there exists a unique global solution of the initial value problem (1.1), (1.2).

Proof. Recall that the equation (1.1) is equivalent to the system (2.8) or (2.9), (2.10). Consider the mappings

$$
\begin{gathered}
\Pi(t, z, u)=\left[P_{1}^{\prime}(t)-G^{-1}(t) Q_{1}(t)\left[A^{\prime}(t)+B(t)\right]\right] P_{1}(t) z+ \\
+G^{-1}(t) Q_{1}(t) f\left(t, P_{1}(t) z+P_{2}(t) u\right), \\
F(t, z, u)=G^{-1}(t) Q_{2}(t)\left[f\left(t, P_{1}(t) z+P_{2}(t) u\right)-A^{\prime}(t) z\right]-u
\end{gathered}
$$


acting from $\left[t_{+}, \infty\right) \times \mathbb{R}^{n} \times \mathbb{R}^{n}$ into $\mathbb{R}^{n}$, and the system

$$
\begin{aligned}
& z^{\prime}(t)=\Pi(t, z(t), u(t)), \\
& F(t, z(t), u(t))=0 .
\end{aligned}
$$

Obviously, the mappings $\Pi(t, z, u), F(t, z, u)$ are continuous in $(t, z, u)$ and have continuous partial derivatives with respect to $z, u$ on $\left[t_{+}, \infty\right) \times \mathbb{R}^{n} \times \mathbb{R}^{n}$. The partial derivatives of $F(t, z, u)$ with respect to $z, u$ have the form

$$
\begin{aligned}
\frac{\partial}{\partial z} F(t, z, u) & =G^{-1}(t)\left[\frac{\partial}{\partial x}\left[Q_{2}(t) f\left(t, P_{1}(t) z+P_{2}(t) u\right)\right]-Q_{2}(t) A^{\prime}(t)\right] P_{1}(t) \\
\frac{\partial}{\partial u} F(t, z, u) & =G^{-1}(t) \frac{\partial}{\partial x}\left[Q_{2}(t) f\left(t, P_{1}(t) z+P_{2}(t) u\right)\right] P_{2}(t)-I_{\mathbb{R}^{n}}= \\
& =G^{-1}(t) \Phi_{t, P_{1}(t) z, P_{2}(t) u} P_{2}(t)-P_{1}(t)
\end{aligned}
$$

where $\Phi_{t, P_{1}(t) z, P_{2}(t) u}$ is the operator (3.2). Denote $\tilde{\Phi}_{t, z, u}=\Phi_{t, P_{1}(t) z, P_{2}(t) u}$.

Lemma 3.1. If a function $x(t)$ is a solution of the equation (1.1) on $\left[t_{0}, t_{1}\right)$ and satisfies the initial condition (1.2), then the functions $z(t)=P_{1}(t) x(t), u(t)=$ $P_{2}(t) x(t)$ are a solution of the system (3.6), (3.7) on $\left[t_{0}, t_{1}\right)$ and satisfy the initial conditions $z\left(t_{0}\right)=P_{1}\left(t_{0}\right) x_{0}, u\left(t_{0}\right)=P_{2}\left(t_{0}\right) x_{0}$, and $z \in C^{1}\left(\left[t_{0}, t_{1}\right), \mathbb{R}^{n}\right), u \in$ $C\left(\left[t_{0}, t_{1}\right), \mathbb{R}^{n}\right)$.

Conversely, if functions $z \in C^{1}\left(\left[t_{0}, t_{1}\right), \mathbb{R}^{n}\right), u \in C\left(\left[t_{0}, t_{1}\right), \mathbb{R}^{n}\right)$ are a solution of the system (3.6), (3.7) on $\left[t_{0}, t_{1}\right)$ and satisfy the initial conditions $z\left(t_{0}\right)=P_{1}\left(t_{0}\right) x_{0}, u\left(t_{0}\right)=P_{2}\left(t_{0}\right) x_{0}$, then $P_{1}(t) z(t)=z(t), P_{2}(t) u(t)=u(t)$ and the function $x(t)=z(t)+u(t)$ is a solution of the equation (1.1) on $\left[t_{0}, t_{1}\right)$ and satisfies the initial condition (1.2).

Proof. Let $x(t)$ be a solution of the equation (1.1) on $\left[t_{0}, t_{1}\right)$ and satisfy (1.2). Notice that $\left(t_{0}, x_{0}\right) \in L_{t_{+}}$since (1.1) is equivalent to the system (2.6), (2.7) and $x\left(t_{0}\right)=x_{0}$ satisfies (2.7) at $t=t_{0}$ (see Remark 2.1). Since (1.1) is equivalent to the system (2.8), then $z(t)=P_{1}(t) x(t), u(t)=P_{2}(t) x(t)$ are a solution of the system $(2.8)$ on $\left[t_{0}, t_{1}\right)$ and consequently are a solution of the system (3.6), (3.7). It is clear that $z\left(t_{0}\right)=P_{1}\left(t_{0}\right) x_{0}$ and $u\left(t_{0}\right)=P_{2}\left(t_{0}\right) x_{0}$. The smoothness of $z(t)$, $u(t)$ follows from the smoothness of $x(t)$ and the projectors $P_{i}(t)$.

Now let $z \in C^{1}\left(\left[t_{0}, t_{1}\right), \mathbb{R}^{n}\right), u \in C\left(\left[t_{0}, t_{1}\right), \mathbb{R}^{n}\right)$ be a solution of the system (3.6), (3.7) on $\left[t_{0}, t_{1}\right)$ and $z\left(t_{0}\right)=P_{1}\left(t_{0}\right) x_{0}, u\left(t_{0}\right)=P_{2}\left(t_{0}\right) x_{0}$. Obviously, $\left(t_{0}, x_{0}\right) \in L_{t_{+}}$. Multiplying (3.7) by $P_{1}(t)$ and $P_{2}(t)$, we get that $P_{1}(t) u(t) \equiv 0$ and $P_{2}(t) u(t) \equiv u(t)$. Multiplying (3.6) by $P_{2}(t)$, we get that $z(t)$ satisfies the equation $P_{2}(t) z^{\prime}(t)=P_{2}(t) P_{1}^{\prime}(t) P_{1}(t) z(t)$. Since $P_{2}(t) z^{\prime}(t)=\left[P_{2}(t) z(t)\right]^{\prime}-P_{2}^{\prime}(t) z(t)$, $P_{2}(t) P_{1}^{\prime}(t)=-P_{2}^{\prime}(t) P_{1}(t)$ and $z\left(t_{0}\right) \in X_{1}\left(t_{0}\right)$, then $P_{2}(t) z(t)$ satisfies the equation $\left[P_{2}(t) z(t)\right]^{\prime}=P_{2}^{\prime}(t)\left[P_{2}(t) z(t)\right]$ and the initial condition $P_{2}\left(t_{0}\right) z\left(t_{0}\right)=0$. Consequently, $P_{2}(t) z(t) \equiv 0$ and therefore $P_{1}(t) z(t) \equiv z(t)$. Thus, the function $x(t)=z(t)+u(t)$ is such that $P_{1}(t) x(t)=z(t)$ and $P_{2}(t) x(t)=u(t)$. Therefore, the function $x(t)=z(t)+u(t)$ is a solution of the system (2.8) on $\left[t_{0}, t_{1}\right)$ and $x\left(t_{0}\right)=x_{0}$. Consequently, it is a solution of $(1.1)$ on $\left[t_{0}, t_{1}\right)$ and satisfies (1.2).

In the proof of Lemma 3.1, it is shown that if $u(t) \in \mathbb{R}^{n}$ satisfies (3.7), then $u(t)=P_{2}(t) u(t)$, i.e., $u(t) \in X_{2}(t)$. 
Lemma 3.2. For each $t \in\left[t_{+}, \infty\right)$ and each $z \in \mathbb{R}^{n}$ there exists a unique $u \in X_{2}(t)$ such that

$$
F(t, z, u)=0 \text {. }
$$

Proof. Notice that $F(t, z, u)=F\left(t, P_{1}(t) z, u\right)$ for any $z \in \mathbb{R}^{n}$, since $Q_{2}(t) A^{\prime}(t)=$ $Q_{2}(t) A^{\prime}(t) P_{1}(t)$, and that the point $\left(t, x_{p_{1}}(t)+x_{p_{2}}(t)\right)$ belongs to $L_{t_{+}}$if and only if $t, x_{p_{1}}(t), x_{p_{2}}(t)$ satisfy $(2.10)$ or the equivalent condition $F\left(t, x_{p_{1}}(t), x_{p_{2}}(t)\right)=0$ (i.e., $t, z(t)=x_{p_{1}}(t), u(t)=x_{p_{2}}(t)$ satisfy (3.7)). Therefore, by virtue of the condition 1$)$, for each $t \in\left[t_{+}, \infty\right)$ and each $z \in \mathbb{R}^{n}$ there exists a unique $u=$ $x_{p_{2}}(t) \in X_{2}(t)$ such that $\left(t, P_{1}(t) z+u\right) \in L_{t_{+}}$, i.e., $F(t, z, u)=0$.

Take any initial point $\left(t_{0}, x_{0}\right) \in L_{t_{+}}$and any fixed $t_{*} \in\left[t_{0}, \infty\right), z_{*} \in \mathbb{R}^{n}$, where $z_{*}=P_{1}\left(t_{*}\right) x_{0}$ for $t_{*}=t_{0}$. By Lemma 3.2, there exists a unique $u_{*} \in X_{2}\left(t_{*}\right)$ $\left(u_{*}=P_{2}\left(t_{*}\right) x_{0}\right.$ for $\left.t_{*}=t_{0}\right)$ such that $F\left(t_{*}, z_{*}, u_{*}\right)=0$. Since the operator $\tilde{\Phi}_{t, z, u}$ is invertible for each point $(t, z, u)=\left(t_{*}, z_{*}, u_{*}\right)$ such that $u_{*} \in X_{2}\left(t_{*}\right)$ and $F\left(t_{*}, z_{*}, u_{*}\right)=0$ (i.e., $\left.\left(t_{*}, P_{1}\left(t_{*}\right) z_{*}+u_{*}\right) \in L_{t_{0}}\right)$, then for such points $(t, z, u)=$ $\left(t_{*}, z_{*}, u_{*}\right)$ the operator

$$
\Psi_{t, z, u}=\frac{\partial}{\partial u} F(t, z, u)=G^{-1}(t) \tilde{\Phi}_{t, z, u} P_{2}(t)-P_{1}(t) \in L\left(\mathbb{R}^{n}\right)
$$

has the inverse $\left[\Psi_{t, z, u}\right]^{-1}=\left[\tilde{\Phi}_{t, z, u}\right]^{-1} G(t) P_{2}(t)-P_{1}(t) \in L\left(\mathbb{R}^{n}\right)$.

Using the implicit function theorems, we obtain the following statement: there exist neighborhoods $U_{\delta_{1}}\left(t_{*}\right)=\left\{t \in\left(t_{0}, \infty\right)|| t-t_{*} \mid<\delta_{1}\right\}$ $\left(U_{\delta_{1}}\left(t_{0}\right)=\left[t_{0}, t_{0}+\delta_{1}\right)\right.$ when $\left.t_{*}=t_{0}\right), U_{\delta_{2}}\left(z_{*}\right) \subset \mathbb{R}^{n}, U_{\delta_{3}}\left(u_{*}\right) \subset \mathbb{R}^{n}$ and a unique function $u=\nu(t, z): U_{\delta_{1}}\left(t_{*}\right) \times U_{\delta_{2}}\left(z_{*}\right) \rightarrow U_{\delta_{3}}\left(u_{*}\right)$ which is continuous in $(t, z)$ and continuously differentiable in $z$ on $U_{\delta_{1}}\left(t_{*}\right) \times U_{\delta_{2}}\left(z_{*}\right)$ and is such that $F(t, z, \nu(t, z))=0$ for $(t, z) \in U_{\delta_{1}}\left(t_{*}\right) \times U_{\delta_{2}}\left(z_{*}\right)$ and $\nu\left(t_{*}, z_{*}\right)=u_{*}$. Since $u=\nu(t, z)$ is a solution of (3.10) (i.e., $F(t, z, \nu(t, z))=0$ ), then $\nu(t, z)=P_{2}(t) \nu(t, z) \in X_{2}(t)$ for each point $(t, z) \in U_{\delta_{1}}\left(t_{*}\right) \times U_{\delta_{2}}\left(z_{*}\right)$. Thus, it is proved that in some neighborhood $U\left(t_{*}, z_{*}\right)$ of each point $\left(t_{*}, z_{*}\right) \in\left[t_{0}, \infty\right) \times \mathbb{R}^{n}$ $\left(z_{*}=P_{1}\left(t_{*}\right) x_{0}\right.$ when $\left.t_{*}=t_{0}\right)$ there exists a unique solution $u=\nu_{t_{*}, z_{*}}(t, z)$ of (3.10), which is continuous in $(t, z)$ and continuously differentiable in $z$, and $\nu_{t_{*}, z_{*}}(t, z) \in X_{2}(t)$ for each $(t, z) \in U\left(t_{*}, z_{*}\right)$. Introduce the function $u=\eta(t, z):\left[t_{0}, \infty\right) \times \mathbb{R}^{n} \rightarrow \mathbb{R}^{n}$ defined by $\eta(t, z)=\nu_{t_{*}, z_{*}}(t, z)$ at the point $(t, z)=\left(t_{*}, z_{*}\right)$ for each $\left(t_{*}, z_{*}\right) \in\left[t_{0}, \infty\right) \times \mathbb{R}^{n}$. Then the function $u=\eta(t, z)$ is continuous in $(t, z)$, continuously differentiable in $z$ and a solution of the equation (3.10) (i.e., $F(t, z, \eta(t, z))=0)$, and $\eta(t, z) \in X_{2}(t)$ for $(t, z) \in\left[t_{0}, \infty\right) \times \mathbb{R}^{n}$. We prove the uniqueness of $u=\eta(t, z)$. Assume that there exists a function $u=\mu(t, z)$ having the same properties as $u=\eta(t, z)$ at some point $(\tilde{t}, \tilde{z}) \in\left[t_{0}, \infty\right) \times \mathbb{R}^{n}$. By Lemma 3.2, there exists a unique $\tilde{u} \in X_{2}(\tilde{t})$ such that $(t, z, u)=(\tilde{t}, \tilde{z}, \tilde{u})$ satisfies $(3.10)$. Consequently, $\eta(\tilde{t}, \tilde{z})=\mu(\tilde{t}, \tilde{z})=\tilde{u}$. It is similarly proved that if the point $(\tilde{t}, \tilde{z})$ belongs to the intersection of neighborhoods $U^{1}\left(t^{1}, z^{1}\right), U^{2}\left(t^{2}, z^{2}\right)$ of some points $\left(t^{1}, z^{1}\right),\left(t^{2}, z^{2}\right) \in\left[t_{0}, \infty\right) \times \mathbb{R}^{n}$ in which the solutions $u=\nu_{t^{1}, z^{1}}(t, z)$ and $u=\nu_{t^{2}, z^{2}}(t, z)$ of (3.10) are defined respectively, then $\nu_{t^{1}, z^{1}}(\tilde{t}, \tilde{z})=\nu_{t^{2}, z^{2}}(\tilde{t}, \tilde{z})=\eta(\tilde{t}, \tilde{z})=\tilde{u}$. This holds for any $(\tilde{t}, \tilde{z}) \in\left[t_{0}, \infty\right) \times \mathbb{R}^{n}$. Consequently, there exists the unique function $u=\eta(t, z)$ with the above properties. 
Substitute the function $u=\eta(t, z)$ in (3.4) and denote $\widetilde{\Pi}(t, z)=\Pi(t, z, \eta(t, z))$. Then the equation (3.6) takes the form

$$
z^{\prime}(t)=\widetilde{\Pi}(t, z(t))
$$

By the properties of $\eta$ and $\Pi$, the function $\widetilde{\Pi}$ is continuous in $(t, z)$ and continuously differentiable in $z$ on $\left[t_{0}, \infty\right) \times \mathbb{R}^{n}$. Hence, there exists a unique solution $z=\zeta(t)$ of (3.12) satisfying the initial condition $\zeta\left(t_{0}\right)=z_{0}$, where $z_{0}=P_{1}\left(t_{0}\right) x_{0}$, on some interval $\left[t_{0}, \alpha\right)$. Notice that $\eta\left(t_{0}, z_{0}\right)=P_{2}\left(t_{0}\right) x_{0}$ and $x_{0}=z_{0}+\eta\left(t_{0}, \zeta_{0}\right)$. The solution $\zeta(t)$ can be continued to a maximal interval of existence and this continuation will be the unique one (see $[18,23]$ ), namely, there exists the maximal interval $\left[t_{0}, \omega\right) \subseteq\left[t_{0}, \infty\right)$ on which there exists a unique solution $z=\zeta(t)$ of (3.12) satisfying the initial condition $\zeta\left(t_{0}\right)=z_{0}$. Since the functions $z=\zeta(t)$ and $u=\gamma(t)$, where $\gamma(t)=\eta(t, \zeta(t))$, are the solution of the system (3.6), (3.7) on $\left[t_{0}, \omega\right)$ and satisfy the initial conditions $\zeta\left(t_{0}\right)=P_{1}\left(t_{0}\right) x_{0}, \gamma\left(t_{0}\right)=P_{2}\left(t_{0}\right) x_{0}$, then, by Lemma 3.1, $\zeta(t)=P_{1}(t) \zeta(t) \in X_{1}(t), \gamma(t)=P_{2}(t) \gamma(t) \in X_{2}(t)$ for all $t \in\left[t_{0}, \omega\right)$, and the function $x(t)=\zeta(t)+\gamma(t)$ is a solution of the equation (1.1) on $\left[t_{0}, \omega\right)$ that satisfies the initial condition (1.2). The uniqueness of the solution $z=\zeta(t), u=\gamma(t)$ of the system (3.6), (3.7) and, accordingly, the solution $x(t)$ of the equation $(1.1)$ on $\left[t_{0}, \omega\right)$ follows from the uniqueness of the solution $u=\eta(t, z)$ of (3.10) and the solution $z=\zeta(t)$ of (3.12). We prove that the maximal interval $\left[t_{0}, \omega\right)$ coincides with $\left[t_{0}, \infty\right)$ (the proof of this statement is similar to the proof of the theorem [16, Ch. 4, Theorem XIII]).

Introduce the positive definite scalar function

$$
V(t, z)=(H(t) z, z)
$$

where $H(t)$ is the operator defined in the condition 3$)\left(V(t, z) \in C^{1}\left(\left[t_{+}, \infty\right) \times\right.\right.$ $\left.\left.\mathbb{R}^{n}, \mathbb{R}\right)\right)$. It is clear that $V(t, z) \rightarrow \infty$ uniformly in $t$ on every finite time interval $[a, b) \subset\left[t_{+}, \infty\right)$ as $\|z\| \rightarrow \infty$. Moreover, since the operator $H(t)$ is positive definite, i.e. there exists a constant $H_{0}>0$ such that $(H(t) z, z) \geq H_{0}\|z\|^{2}$ for all $z \in \mathbb{R}^{n}, t \in\left[t_{+}, \infty\right)$, then $V(t, z) \rightarrow \infty$ uniformly in $t$ on $\left[t_{+}, \infty\right)$ as $\|z\| \rightarrow \infty$. The gradient of the function $V$ has the form $\frac{\partial V}{\partial z}(t, z)=2 z^{T} H(t)$. The derivative of $V$ along the trajectories of the system (3.12) has the form

$$
V_{(3.12)}^{\prime}(t, z)=\left(H^{\prime}(t) z, z\right)+2(H(t) z, \widetilde{\Pi}(t, z)) .
$$

The solution $z=\zeta(t)$ of (3.12) either is defined in the future, i.e., the maximal interval of existence of the solution coincides with $\left[t_{0}, \infty\right)$, or has the finite escape time $\left[t_{0}, \omega\right)$, i.e., $\omega<\infty$ and $\lim _{t \rightarrow \omega-0}\|\zeta(t)\|=\infty$, [16]. Notice that $\zeta(t)=P_{1}(t) x(t), \eta(t, \zeta(t))=P_{2}(t) x(t)$, where $x(t)=\zeta(t)+\eta(t, \zeta(t))$ is a solution of (1.1). Assume that the solution $z=\zeta(t)$ has the finite escape time. Then there exists $t_{1} \in\left(t_{0}, \omega\right)$ such that $\zeta(t)$ is contained in the set $B_{R_{0}}^{c}=\left\{x \in \mathbb{R}^{n} \mid(t, x) \in L_{t_{0}},\left\|P_{1}(t) x\right\| \geq R_{0}\right\}$, where $R_{0}>R(R>0$ is defined 
in the condition 3$)$ ), for any $t \in\left[t_{1}, \omega\right)$. By the condition 3$)$, the inequality

$$
\begin{gathered}
V_{(3.12)}^{\prime}(t, \zeta(t))=\left(H^{\prime}(t) \zeta(t), \zeta(t)\right)+2(H(t) \zeta(t), \widetilde{\Pi}(t, \zeta(t)))= \\
=\left(H^{\prime}(t) P_{1}(t) x(t), P_{1}(t) x(t)\right)+2\left(H(t) P_{1}(t) x(t),\left[P_{1}^{\prime}(t)-\right.\right. \\
\left.\left.-G^{-1}(t) Q_{1}(t)\left[A^{\prime}(t)+B(t)\right]\right] P_{1}(t) x(t)+G^{-1}(t) Q_{1}(t) f\left(t, P_{1}(t) x(t)+P_{2}(t) x(t)\right)\right)= \\
=2 J\left(t, P_{1}(t) x(t)\right) \leq 2 k(t) U((H(t) \zeta(t), \zeta(t)))=2 k(t) U(V(t, \zeta(t))) .
\end{gathered}
$$

holds for $t \geq t_{1}$. Therefore, for $t \geq t_{1}$ the function $v(t)=V(t, \zeta(t))$ is a positive solution of the differential inequality

$$
v^{\prime} \leq 2 k(t) U(v)
$$

By assumption, the solution $\zeta(t)$ has the finite escape time, therefore $v(t)$ has the finite escape time. On the other hand, from the properties of the functions $k(t), U(v)$ it follows that the inequality (3.16) $\left(t \geq t_{+}\right)$has no positive solutions with finite escape time, which contradicts the assumption. Consequently, $\zeta(t)$ is defined in the future, i.e., $\left[t_{0}, \omega\right)=\left[t_{0}, \infty\right)$.

Thus, it is proved that the function $x(t)=\zeta(t)+\gamma(t)$, where $\gamma(t)=\eta(t, \zeta(t))$, is a unique solution of the initial value problem $(1.1),(1.2)$ on $\left[t_{0}, \infty\right)$. Consequently, for each initial point $\left(t_{0}, x_{0}\right) \in L_{t_{+}}$there exists a unique global solution of (1.1), (1.2).

The following are the definitions introduced in [21].

A system of $s$ pairwise disjoint projectors $\left\{\Theta_{k}\right\}_{k=1}^{s}$ (the projectors are onedimensional), $\Theta_{k} \in L(Z)$, the sum of which is the identity operator $I_{Z}$ in an $s$-dimensional linear space $Z$, i.e., $\Theta_{i} \Theta_{j}=\delta_{i j} \Theta_{i}$ and $I_{Z}=\sum_{k=1}^{s} \Theta_{k}$, is called an additive resolution of the identity in $Z$. The additive resolution of the identity generates the decomposition $Z=Z_{1} \dot{+} \cdots \dot{+} Z_{s}$ into the direct sum of the onedimensional subspaces $Z_{k}=\Theta_{k} Z$, and the system $\left\{z_{k} \in Z\right\}_{k=1}^{s}$ of the vectors such that $z_{k} \neq 0$ and $z_{k}=\Theta_{k} z_{k}$ forms a basis of $Z$. Note that the property of basis invertibility does not depend on the choice of an additive resolution of the identity or a basis of $Z$.

Definition 3.1. An operator function $\Phi: D \rightarrow L(W, Z)$, where $W, Z$ are $s$ dimensional linear spaces and $D \subset W$, is called basis invertible on an interval $J \subset D$, if for some additive resolution of the identity $\left\{\Theta_{k}\right\}_{k=1}^{s}$ in the space $Z$ and for any set $\left\{w^{k}\right\}_{k=1}^{s}$ of elements $w^{k} \in J$ the operator $\Lambda=\sum_{k=1}^{s} \Theta_{k} \Phi\left(w^{k}\right) \in L(W, Z)$ has the inverse $\Lambda^{-1} \in L(Z, W)$.

Obviously, it follows from the basis invertibility of the mapping $\Phi$ on the interval $J$ that $\Phi$ is invertible on this interval, i.e., for each point $w^{*} \in J$ its image under the mapping $\Phi$ is the invertible operator $\Phi\left(w^{*}\right) \in L(W, Z)$. The converse is not true (see [8, Example 2.1]) unless the spaces $W, Z$ are one-dimensional.

A theorem similar to Theorem 3.1, in which there is no the requirement of the uniqueness of $x_{p_{2}}(t)$ in the condition 1$)$, but in the condition 2) the requirement of invertibility is replaced by the requirement of basis invertibility, is proved below. 
Remark 3.1. Notice that $\operatorname{rk} P_{j}(t)=\operatorname{rk} Q_{j}(t)=\operatorname{dim} X_{j}(t)=\operatorname{dim} Y_{j}(t), j=1,2$, $\operatorname{dim} Y_{1}(t)=\operatorname{rk} A(t)$ and that, by virtue of the smoothness of the projectors (i.e., projection operator functions) $P_{j}(t), Q_{j}(t)$, the dimensions of the subspaces $X_{j}(t)=P_{j}(t) \mathbb{R}^{n}, Y_{j}(t)=Q_{j}(t) \mathbb{R}^{n}, j=1,2$, are constant for all $t \in\left[t_{+}, \infty\right)$ (see [12, p. 34, Lemma 4.10]). We denote $\operatorname{dim} X_{2}(t)=\operatorname{dim} Y_{2}(t)=d$, then $\operatorname{dim} X_{1}(t)=\operatorname{dim} Y_{1}(t)=n-d\left(t \in\left[t_{+}, \infty\right)\right)$.

Theorem 3.2. Let $f \in C\left(\left[t_{+}, \infty\right) \times \mathbb{R}^{n}, \mathbb{R}^{n}\right), \frac{\partial}{\partial x} f \in C\left(\left[t_{+}, \infty\right) \times \mathbb{R}^{n}, L\left(\mathbb{R}^{n}\right)\right)$, $A, B \in C^{1}\left(\left[t_{+}, \infty\right), L\left(\mathbb{R}^{n}\right)\right)$, the pencil $\lambda A(t)+B(t)$ satisfy (2.1), where $C_{2} \in C^{1}\left(\left[t_{+}, \infty\right),(0, \infty)\right)$, and the following conditions be satisfied:

1) for each $t \in\left[t_{+}, \infty\right)$ and each $x_{p_{1}}(t) \in X_{1}(t)$ there exists $x_{p_{2}}(t) \in X_{2}(t)$ such that (3.1);

2) for any fixed $t_{*} \in\left[t_{+}, \infty\right), x_{p_{1}}^{*}\left(t_{*}\right) \in X_{1}\left(t_{*}\right), x_{p_{2}}^{i}\left(t_{*}\right) \in X_{2}\left(t_{*}\right)$ such that $\left(t_{*}, x_{p_{1}}^{*}\left(t_{*}\right)+x_{p_{2}}^{i}\left(t_{*}\right)\right) \in L_{t_{+}}, i=1,2$, the operator function

$$
\begin{aligned}
\Phi_{t_{*}, x_{p_{1}}^{*}\left(t_{*}\right)}: X_{2}\left(t_{*}\right) \rightarrow L\left(X_{2}\left(t_{*}\right), Y_{2}\left(t_{*}\right)\right), \\
\Phi_{t_{*}, x_{p_{1}}^{*}\left(t_{*}\right)}\left(x_{p_{2}}\left(t_{*}\right)\right)=\left[\frac{\partial}{\partial x}\left[Q_{2}\left(t_{*}\right) f\left(t_{*}, x_{p_{1}}^{*}\left(t_{*}\right)+x_{p_{2}}\left(t_{*}\right)\right)\right]-B\left(t_{*}\right)\right] P_{2}\left(t_{*}\right),
\end{aligned}
$$

is basis invertible on $\left[x_{p_{2}}^{1}\left(t_{*}\right), x_{p_{2}}^{2}\left(t_{*}\right)\right]$;

3) it coincides with the condition 3) of Theorem 3.1.

Then for each initial point $\left(t_{0}, x_{0}\right) \in L_{t_{+}}$there exists a unique global solution of the initial value problem (1.1), (1.2).

Proof. As in the proof of Theorem 3.1, we consider the mappings (3.4), (3.5) and the system (3.6), (3.7). The mapping (3.5) has continuous partial derivatives with respect to $z, u$ on $\left[t_{+}, \infty\right) \times \mathbb{R}^{n} \times \mathbb{R}^{n}$. The partial derivative with respect to $u$ have the form $\frac{\partial}{\partial u} F(t, z, u)=G^{-1}(t) \frac{\partial}{\partial x}\left[Q_{2}(t) f\left(t, P_{1}(t) z+P_{2}(t) u\right)\right] P_{2}(t)-$ $I_{\mathbb{R}^{n}}=G^{-1}(t) \Phi_{t, P_{1}(t) z}\left(P_{2}(t) u\right) P_{2}(t)-P_{1}(t)$, where $\Phi_{t, P_{1}(t) z}\left(P_{2}(t) u\right)$ is the operator function (3.17). Denote $\tilde{\Phi}_{t, z}(u)=\Phi_{t, P_{1}(t) z}\left(P_{2}(t) u\right)$ and introduce the operator function

$$
\Psi_{t, z}: \mathbb{R}^{n} \rightarrow L\left(\mathbb{R}^{n}\right), \quad \Psi_{t, z}(u)=\frac{\partial}{\partial u} F(t, z, u)=G^{-1}(t) \tilde{\Phi}_{t, z}(u) P_{2}(t)-P_{1}(t) .
$$

By virtue of the basis invertibility of (3.17) for any fixed $t_{*} \in\left[t_{+}, \infty\right), z_{*} \in \mathbb{R}^{n}$, $u_{*}^{i} \in X_{2}\left(t_{*}\right)(i=1,2)$ such that $F\left(t_{*}, z_{*}, u_{*}^{i}\right)=0$ (i.e., $\left.\left(t_{*}, P_{1}\left(t_{*}\right) z_{*}+u_{*}^{i}\right) \in L_{t_{+}}\right)$, the operator function $\tilde{\Phi}_{t_{*}, z_{*}}$ is basis invertible on $\left[u_{*}^{1}, u_{*}^{2}\right]$. This property is needed to prove Lemma 3.2 (see below). It also follows from the basis invertibility of $\tilde{\Phi}_{t_{*}, z_{*}}(u)$ that there exists the inverse operator $\left[\tilde{\Phi}_{t_{*}, z_{*}}\left(u_{*}\right)\right]^{-1}$ for any fixed $t_{*} \in$ $\left[t_{+}, \infty\right), z_{*} \in \mathbb{R}^{n}, u_{*} \in X_{2}\left(t_{*}\right)$ such that $F\left(t_{*}, z_{*}, u_{*}\right)=0$ and, consequently, for such points $\left(t_{*}, z_{*}, u_{*}\right)$ the operator $\Psi_{t_{*}, z_{*}}\left(u_{*}\right)$ has the inverse $\left[\Psi_{t_{*}, z_{*}}\left(u_{*}\right)\right]^{-1}=$ $\left[\tilde{\Phi}_{t_{*}, z_{*}}\left(u_{*}\right)\right]^{-1} G\left(t_{*}\right) P_{2}\left(t_{*}\right)-P_{1}\left(t_{*}\right) \in L\left(\mathbb{R}^{n}\right)$.

Lemmas 3.1 and 3.2 remain valid, however, the proof of Lemma 3.2 changes.

Lemma (Lemma 3.2). For each $t \in\left[t_{+}, \infty\right)$ and each $z \in \mathbb{R}^{n}$ there exists a unique $u \in X_{2}(t)$ such that (3.10). 
Proof. By virtue of the condition 1) (here the existence of a unique $x_{p_{2}}(t)$ is not required, unlike the condition 1) of Theorem 3.1), for each $t \in\left[t_{+}, \infty\right)$ and each $z \in \mathbb{R}^{n}$ there exists $u \in X_{2}(t)$ such that $\left(t, P_{1}(t) z+u\right) \in L_{t_{+}}$, i.e., $F(t, z, u)=0$. We prove the uniqueness of such $u$. Consider arbitrary fixed $t_{*} \in\left[t_{+}, \infty\right), z_{*} \in \mathbb{R}^{n}$, $u_{*}^{i} \in X_{2}\left(t_{*}\right), i=1,2$, such that $F\left(t_{*}, z_{*}, u_{*}^{i}\right)=0$. The basis invertibility of $\tilde{\Phi}_{t_{*}, z_{*}}(u)$ on $\left[u_{*}^{1}, u_{*}^{2}\right]$ means that for any set of points $\left\{u_{k}\right\}_{k=1}^{d} \subset\left[u_{*}^{1}, u_{*}^{2}\right]$, the operator

$$
\Lambda_{1}=\sum_{k=1}^{d} \tilde{\Theta}_{k}\left(t_{*}\right) \tilde{\Phi}_{t_{*}, z_{*}}\left(u_{k}\right) \in L\left(X_{2}\left(t_{*}\right), Y_{2}\left(t_{*}\right)\right),
$$

where $\left\{\tilde{\Theta}_{k}\left(t_{*}\right)\right\}_{k=1}^{d}$ is some additive resolution of the identity in $Y_{2}\left(t_{*}\right)$ $\left(d=\operatorname{dim} Y_{2}(t)=\operatorname{dim} X_{2}(t), t \in\left[t_{+}, \infty\right)\right.$, see Remark 3.1), has the inverse $\Lambda_{1}^{-1} \in L\left(Y_{2}\left(t_{*}\right), X_{2}\left(t_{*}\right)\right)$. Since $Q_{2}\left(t_{*}\right)$ (restricted to $\left.Y_{2}\left(t_{*}\right)\right)$ is the identity in $Y_{2}\left(t_{*}\right)$ (because $Q_{2}\left(t_{*}\right) y_{*}=y_{*}$ for any $y_{*} \in Y_{2}\left(t_{*}\right)$ ), then we choose $\left\{\tilde{\Theta}_{k}\left(t_{*}\right)\right\}_{k=1}^{d}$ such that $\sum_{k=1}^{d} \tilde{\Theta}_{k}\left(t_{*}\right)=\left.Q_{2}\left(t_{*}\right)\right|_{Y_{2}\left(t_{*}\right)}$, i.e., $\left\{\tilde{\Theta}_{k}\left(t_{*}\right)\right\}_{k=1}^{d}$ is an additive resolution of the identity $\left.Q_{2}\left(t_{*}\right)\right|_{Y_{2}\left(t_{*}\right)}$ in $Y_{2}\left(t_{*}\right)$. Then the system $\left\{\Theta_{k}\left(t_{*}\right)\right\}_{k=1}^{d}$ of the projectors $\Theta_{k}\left(t_{*}\right)=\left.G^{-1}\left(t_{*}\right) \tilde{\Theta}_{k}\left(t_{*}\right) G\left(t_{*}\right)\right|_{X_{2}\left(t_{*}\right)}$ is an additive resolution of the identity $\left.P_{2}\left(t_{*}\right)\right|_{X_{2}\left(t_{*}\right)}$ in $X_{2}\left(t_{*}\right) \quad\left(\sum_{k=1}^{d} \Theta_{k}\left(t_{*}\right)=\left.P_{2}\left(t_{*}\right)\right|_{X_{2}\left(t_{*}\right)}\right)$. Note that $F\left(t_{*}, z_{*}, u_{*}\right)=P_{2}\left(t_{*}\right) F\left(t_{*}, z_{*}, u_{*}\right)$ for any $t_{*} \in\left[t_{+}, \infty\right), z_{*} \in \mathbb{R}^{n}, u_{*} \in X_{2}\left(t_{*}\right)$. The projections $F_{k}\left(t_{*}, z_{*}, u_{*}\right)=\Theta_{k}\left(t_{*}\right) F\left(t_{*}, z_{*}, u_{*}\right)=\Theta_{k}\left(t_{*}\right) P_{2}\left(t_{*}\right) F\left(t_{*}, z_{*}, u_{*}\right)$, where $u_{*} \in X_{2}\left(t_{*}\right)$, are the functions with values in the one-dimensional spaces $\Theta_{k}\left(t_{*}\right) X_{2}\left(t_{*}\right)$ isomorphic to $\mathbb{R}$. By the formula of finite increments, there exists a point $u_{k} \in\left[u_{*}^{1}, u_{*}^{2}\right]$ such that $F_{k}\left(t_{*}, z_{*}, u_{*}^{2}\right)-F_{k}\left(t_{*}, z_{*}, u_{*}^{1}\right)=\frac{\partial}{\partial u} F_{k}\left(t_{*}, z_{*}, u_{k}\right)\left(u_{*}^{2}-\right.$ $\left.u_{*}^{1}\right)=\Theta_{k}\left(t_{*}\right) P_{2}\left(t_{*}\right) \frac{\partial}{\partial u} F\left(t_{*}, z_{*}, u_{k}\right)\left(u_{*}^{2}-u_{*}^{1}\right)=\Theta_{k}\left(t_{*}\right) P_{2}\left(t_{*}\right) \Psi_{t_{*}, z_{*}}\left(u_{k}\right)\left(u_{*}^{2}-u_{*}^{1}\right)$, $k=\overline{1, d}$. By summing the obtained expressions over $k$ and taking into account that $F\left(t_{*}, z_{*}, u_{*}^{i}\right)=0(i=1,2)$, we obtain that $\sum_{k=1}^{d} \Theta_{k}\left(t_{*}\right) P_{2}\left(t_{*}\right) \Psi_{t_{*}, z_{*}}\left(u_{k}\right)\left(u_{*}^{2}-\right.$ $\left.u_{*}^{1}\right)=G^{-1}\left(t_{*}\right) \sum_{k=1}^{d} \tilde{\Theta}_{k}\left(t_{*}\right) \tilde{\Phi}_{t_{*}, z_{*}}\left(u_{k}\right)\left(u_{*}^{2}-u_{*}^{1}\right)=G^{-1}\left(t_{*}\right) \Lambda_{1}\left(u_{*}^{2}-u_{*}^{1}\right)=0$. Since there exists $\Lambda_{1}^{-1}$, then $u_{*}^{2}=u_{*}^{1}$.

The further proof of the theorem coincides with the proof of Theorem 3.1 (see the part of the proof which is located below Lemma 3.2).

\subsection{Lagrange stability and instability.}

Theorem 3.3. Let the conditions of Theorem 3.1 or Theorem 3.2 be fulfilled, $\int_{t_{+}}^{\infty} k(t) d t<\infty$, and one of the following conditions be also fulfilled:

$\sup _{(t, x) \in L_{t_{+}},\left\|P_{1}(t) x\right\| \leq M, M=\text { const }>0}\left\|G^{-1}(t) Q_{2}(t)\left[f(t, x)-A^{\prime}(t) P_{1}(t) x\right]\right\|<\infty ;$

b) for any point $\left(t, x_{p_{1}}(t)+x_{p_{2}}(t)\right) \in L_{t_{+}}$such that $\left\|x_{p_{1}}(t)\right\| \leq M$, $M=$ const $>0$, there exists a constant $K_{M}>0$, independent of $t, x_{p_{2}}(t)$, such that $\left\|x_{p_{2}}(t)\right\| \leq K_{M}$; 
c) for each $t_{*} \in\left[t_{+}, \infty\right)$ there exists $\tilde{x}_{p_{2}}\left(t_{*}\right) \in X_{2}\left(t_{*}\right)$ such that for any fixed $x_{p_{1}}^{*}\left(t_{*}\right) \in X_{1}\left(t_{*}\right), x_{p_{2}}^{*}\left(t_{*}\right) \in X_{2}\left(t_{*}\right)$, satisfying $\left(t_{*}, x_{p_{1}}^{*}\left(t_{*}\right)+x_{p_{2}}^{*}\left(t_{*}\right)\right) \in L_{t_{+}}$, the operator function (3.17) is basis invertible on $\left(\tilde{x}_{p_{2}}\left(t_{*}\right), x_{p_{2}}^{*}\left(t_{*}\right)\right]$ and the corresponding inverse operator is bounded uniformly in $t_{*}, x_{p_{2}}\left(t_{*}\right)$ (i.e., the operator $\Lambda_{1}^{-1}=\left[\sum_{k=1}^{d} \tilde{\Theta}_{k}\left(t_{*}\right) \Phi_{t_{*}, x_{p_{1}}^{*}\left(t_{*}\right)}\left(x_{p_{2}, k}\left(t_{*}\right)\right)\right]^{-1}$ inverse to the operator (3.19), where $z_{*}=x_{p_{1}}^{*}\left(t_{*}\right)$ and $\left\{u_{k}=x_{p_{2}, k}\left(t_{*}\right)\right\}_{k=1}^{d}$ is an arbitrary set of the elements from $\left(\tilde{x}_{p_{2}}\left(t_{*}\right), x_{p_{2}}^{*}\left(t_{*}\right)\right]$, is bounded uniformly in $t_{*}, x_{p_{2}, k}\left(t_{*}\right)$ on $\left[t_{+}, \infty\right)$, $\left.\left(\tilde{x}_{p_{2}}\left(t_{*}\right), x_{p_{2}}^{*}\left(t_{*}\right)\right]\right)$, and also $\sup _{t_{*} \in\left[t_{+}, \infty\right)}\left\|\tilde{x}_{p_{2}}\left(t_{*}\right)\right\|<\infty$ and

$$
\begin{aligned}
& \sup _{t \in\left[t_{+}, \infty\right),\left\|x_{p_{1}}(t)\right\| \leq M, M=\text { const }>0} \| G^{-1}(t) Q_{2}(t)\left[f\left(t, x_{p_{1}}(t)+\tilde{x}_{p_{2}}\left(t_{*}\right)\right)-\right. \\
& \left.-A^{\prime}(t) x_{p_{1}}(t)\right] \|<\infty \text {. }
\end{aligned}
$$

Then the equation (1.1) is Lagrange stable.

Proof. Just as for Theorem 3.1, we prove that $z=\zeta(t)$ and $u=\gamma(t)$, where $\gamma(t)=$ $\eta(t, \zeta(t))$, are the unique solution of the system (3.6), (3.7) on $\left[t_{0}, \infty\right)$ satisfying the initial conditions $\zeta\left(t_{0}\right)=P_{1}\left(t_{0}\right) x_{0}, \gamma\left(t_{0}\right)=P_{2}\left(t_{0}\right) x_{0}$, where $\left(t_{0}, x_{0}\right) \in L_{t_{+}}$, and that $x(t)=\zeta(t)+\gamma(t)$ is the unique solution of the initial value problem (1.1), (1.2). Recall that $\zeta(t)=P_{1}(t) x(t), \gamma(t)=P_{2}(t) x(t)\left(\zeta(t) \in X_{1}(t), \gamma(t) \in X_{2}(t)\right)$ and $(t, \zeta(t)+\gamma(t)) \in L_{t_{0}}$ for any $t \in\left[t_{0}, \infty\right)$.

It follows from the properties of the functions $k(t), U(v)$ that the inequality (3.16) $\left(t \geq t_{+}\right)$has no unbounded positive solution for $t \geq t_{+}$. Consequently, the solution $z=\zeta(t)$ is bounded on $\left[t_{0}, \infty\right)$, i.e., there exists a constant $M_{*}>0$ such that

$$
\|\zeta(t)\| \leq M_{*} \text { for all } t \in\left[t_{0}, \infty\right) .
$$

Since the equation (3.7) can be rewritten in the form $u(t)=$ $G^{-1}(t) Q_{2}(t)\left[f\left(t, P_{1}(t) z(t)+P_{2}(t) u(t)\right)-A^{\prime}(t) z(t)\right]$, then

$$
\gamma(t)=\eta(t, \zeta(t))=G^{-1}(t) Q_{2}(t)\left[f(t, \zeta(t)+\eta(t, \zeta(t)))-A^{\prime}(t) \zeta(t)\right]
$$

Hence, by (3.21) and the condition a), there exists a constant $K_{M_{*}}=K\left(M_{*}\right)>0$ such that

$$
\|\gamma(t)\|=\|\eta(t, \zeta(t))\| \leq K_{M_{*}}<\infty \text { for all } t \in\left[t_{0}, \infty\right)
$$

Also, by (3.21) and the condition b), there exists a constant $K_{M_{*}}>0$ such that (3.23).

Now we prove the boundedness of $\|\gamma(t)\|$ using the condition c).

Take arbitrary fixed $t_{*} \in\left[t_{+}, \infty\right), z_{*} \in \mathbb{R}^{n}, u_{*} \in X_{2}\left(t_{*}\right)$ satisfying the condition $F\left(t_{*}, z_{*}, u_{*}\right)=0$ (i.e., $\left.\left(t_{*}, P_{1}\left(t_{*}\right) z_{*}+u_{*}\right) \in L_{t_{+}}\right)$. By virtue of the condition $\mathrm{c})$, there exists an element $\tilde{u}_{*}=\tilde{u}\left(t_{*}\right) \in X_{2}\left(t_{*}\right)$ such 
that the operator function $\tilde{\Phi}_{t_{*}, z_{*}}(u)=\Phi_{t_{*}, P_{1}\left(t_{*}\right) z_{*}}\left(P_{2}\left(t_{*}\right) u\right)(3.17)$ is basis invertible on $\left(\tilde{u}_{*}, u_{*}\right]$ and the corresponding inverse operator, i.e., the operator $\Lambda_{1}^{-1}=\left[\sum_{k=1}^{d} \tilde{\Theta}_{k}\left(t_{*}\right) \tilde{\Phi}_{t_{*}, z_{*}}\left(u_{k}\right)\right]^{-1}=\Lambda_{1}^{-1}\left(t_{*}, z_{*}, u_{k}\right) \in L\left(Y_{2}\left(t_{*}\right), X_{2}\left(t_{*}\right)\right)$ inverse to the operator (3.19), where $\left\{u_{k}\right\}_{k=1}^{d}$ is an arbitrary set of the elements $u_{k} \in\left(\tilde{u}_{*}, u_{*}\right]\left(d=\operatorname{dim} X_{2}\left(t_{*}\right)\right)$ and $\left\{\tilde{\Theta}_{k}\left(t_{*}\right)\right\}_{k=1}^{d}$ is an additive resolution of the identity in $Y_{2}\left(t_{*}\right)$, is bounded uniformly in $t_{*}, u_{k}$ on $\left[t_{+}, \infty\right)$, $\left(\tilde{u}_{*}, u_{*}\right]$. As in the proof of Lemma (see p. 255), we choose $\left\{\tilde{\Theta}_{k}\left(t_{*}\right)\right\}_{k=1}^{d}$ such that $\sum_{k=1}^{d} \tilde{\Theta}_{k}\left(t_{*}\right)=\left.Q_{2}\left(t_{*}\right)\right|_{Y_{2}\left(t_{*}\right)}$ and take the additive resolution of the $\left\{\Theta_{k}\left(t_{*}\right)=\left.G^{-1}\left(t_{*}\right) \tilde{\Theta}_{k}\left(t_{*}\right) G\left(t_{*}\right)\right|_{X_{2}\left(t_{*}\right)}\right\}_{k=1}^{d}$ in $X_{2}\left(t_{*}\right)$. Also, consider the projections $F_{k}\left(t_{*}, z_{*}, u\right)=\Theta_{k}\left(t_{*}\right) F\left(t_{*}, z_{*}, u\right)=\Theta_{k}\left(t_{*}\right) P_{2}\left(t_{*}\right) F\left(t_{*}, z_{*}, u\right)$, where $u \in X_{2}\left(t_{*}\right)$. By the formula of finite increments, there exists a point $u_{k} \in\left(\tilde{u}_{*}, u_{*}\right]$ such that $F_{k}\left(t_{*}, z_{*}, u_{*}\right)-F_{k}\left(t_{*}, z_{*}, \tilde{u}_{*}\right)=\frac{\partial}{\partial u} F_{k}\left(t_{*}, z_{*}, u_{k}\right)\left(u_{*}-\tilde{u}_{*}\right)=$ $\Theta_{k}\left(t_{*}\right) P_{2}\left(t_{*}\right) \Psi_{t_{*}, z_{*}}\left(u_{k}\right)\left(u_{*}-\tilde{u}_{*}\right)$, where the operator function $\Psi_{t, z}$ is defined in $(3.18), k=\overline{1, d}$. Since $F_{k}\left(t_{*}, z_{*}, u_{*}\right)=0$, then, by summing the obtained equalities over $k$, we obtain that there exists a set $\left\{u_{k}\right\}_{k=1}^{d} \subset\left(\tilde{u}_{*}, u_{*}\right]$ such that $-F\left(t_{*}, z_{*}, \tilde{u}_{*}\right)=G^{-1}\left(t_{*}\right) \Lambda_{1}\left(u_{*}-\tilde{u}_{*}\right)$. Since there exists $\Lambda_{1}^{-1}$, then $u_{*}=\tilde{u}_{*}-\Lambda_{1}^{-1} G\left(t_{*}\right) F\left(t_{*}, z_{*}, \tilde{u}_{*}\right)=\tilde{u}_{*}-\Lambda_{1}^{-1}\left(Q_{2}\left(t_{*}\right)\left[f\left(t_{*}, P_{1}\left(t_{*}\right) z_{*}+\right.\right.\right.$ $\left.\left.\left.P_{2}\left(t_{*}\right) \tilde{u}_{*}\right)-A^{\prime}\left(t_{*}\right) z_{*}\right]-G\left(t_{*}\right) \tilde{u}_{*}\right)$. This holds for any fixed $t_{*} \in\left[t_{+}, \infty\right)$, $z_{*} \in \mathbb{R}^{n}, u_{*} \in X_{2}\left(t_{*}\right)$ satisfying $F\left(t_{*}, z_{*}, u_{*}\right)=0$. Therefore, for each $t_{*} \in\left[t_{0}, \infty\right)$ the equality $\gamma\left(t_{*}\right)=\eta\left(t_{*}, \zeta\left(t_{*}\right)\right)=\tilde{u}_{*}-\Lambda_{1}^{-1} G\left(t_{*}\right) F\left(t_{*}, z_{*}, \tilde{u}_{*}\right)=\tilde{u}_{*}-$ $\Lambda_{1}^{-1} G\left(t_{*}\right)\left(G^{-1}\left(t_{*}\right) Q_{2}\left(t_{*}\right)\left[f\left(t_{*}, \zeta\left(t_{*}\right)+P_{2}\left(t_{*}\right) \tilde{u}_{*}\right)-A^{\prime}\left(t_{*}\right) \zeta\left(t_{*}\right)\right]-\tilde{u}_{*}\right)$ is fulfilled. By virtue of the condition $\mathrm{c})$, the set of the elements $\tilde{u}_{*}=\tilde{u}\left(t_{*}\right)$ is bounded, i.e., there exists a constant $\tilde{M}<\infty$ such that $\left\|\tilde{u}_{*}\right\|=\left\|\tilde{u}\left(t_{*}\right)\right\| \leq \tilde{M}$ for each $t_{*} \in\left[t_{+}, \infty\right)$. From the continuity of the nonlinear mapping $\Lambda_{1}^{-1}=\Lambda_{1}^{-1}\left(t_{*}, z_{*}, u_{k}\right)$ in $t_{*}, z_{*}$ and the compactness of the ball $\left\|\zeta\left(t_{*}\right)\right\| \leq M_{*}, t_{*} \in\left[t_{0}, \infty\right)$, where $z=\zeta(t) \in C\left(\left[t_{0}, \infty\right), \mathbb{R}^{n}\right)\left(\zeta(t) \in X_{1}(t)\right)$, it follows that $\Lambda_{1}^{-1}$ is uniformly continuous in $z_{*}$ (in $P_{1}\left(t_{*}\right) z_{*}$ ) and is bounded on $\left\|z_{*}\right\|=\left\|\zeta\left(t_{*}\right)\right\| \leq M_{*}$. By the condition c), $\Lambda_{1}^{-1}=\Lambda_{1}^{-1}\left(t_{*}, z_{*}, u_{k}\right) \in L\left(Y_{2}\left(t_{*}\right), X_{2}\left(t_{*}\right)\right)$ is bounded uniformly in $t_{*}, u_{k}$ on $\left[t_{+}, \infty\right),\left(\tilde{u}_{*}, u_{*}\right]$. Therefore, there exists a constant $N>0$, independent of $t_{*}, z_{*}, u^{k}$, such that $\left\|\Lambda_{1}^{-1}\right\| \leq N$ for each $t_{*} \in\left[t_{+}, \infty\right)$, each $z_{*} \in \mathbb{R}^{n}$ and each $u_{*} \in X_{2}\left(t_{*}\right)$ satisfying $F\left(t_{*}, z_{*}, u_{*}\right)=0$ and for any set $\left\{u_{k}\right\}_{k=1}^{d} \subset\left(\tilde{u}_{*}, u_{*}\right]$. Thus, $\left\|\gamma\left(t_{*}\right)\right\| \leq \tilde{M}\left(1+N\left\|G\left(t_{*}\right)\right\|\right)+\| G^{-1}\left(t_{*}\right) Q_{2}\left(t_{*}\right)\left[f\left(t_{*}, \zeta\left(t_{*}\right)+P_{2}\left(t_{*}\right) \tilde{u}_{*}\right)-\right.$ $\left.A^{\prime}\left(t_{*}\right) \zeta\left(t_{*}\right)\right] \|$ for each $t_{*} \in\left[t_{+}, \infty\right)$. Then it follows from (3.21), (3.20) that there exists a constant $K_{M_{*}}>0$ such that $\left\|\gamma\left(t_{*}\right)\right\| \leq K_{M_{*}}$ for all $t_{*} \in\left[t_{+}, \infty\right)$.

It follows from the above that $\|x(t)\|=\|\zeta(t)+\gamma(t)\| \leq M_{*}+K_{M_{*}}$ for all $t \in\left[t_{0}, \infty\right)$, i.e., the solution $x(t)$ is bounded on $\left[t_{0}, \infty\right)$ and therefore Lagrange stable. Since for every consistent initial point $\left(t_{0}, x_{0}\right)$ (i.e., for $\left.\left(t_{0}, x_{0}\right) \in L_{t_{+}}\right)$there exists a unique solution of the initial value problem (1.1), (1.2) which is Lagrange stable, then every solution of (1.1), (1.2) is Lagrange stable (recall that the initial value problem $(1.1),(1.2)$ has a solution only for the initial points $\left.\left(t_{0}, x_{0}\right) \in L_{t_{+}}\right)$. Thus, the equation (1.1) is Lagrange stable.

Theorem 3.4. Let $f \in C\left(\left[t_{+}, \infty\right) \times \mathbb{R}^{n}, \mathbb{R}^{n}\right), \frac{\partial}{\partial x} f \in C\left(\left[t_{+}, \infty\right) \times \mathbb{R}^{n}, L\left(\mathbb{R}^{n}\right)\right)$, $A, B \in C^{1}\left(\left[t_{+}, \infty\right), L\left(\mathbb{R}^{n}\right)\right)$ and the pencil $\lambda A(t)+B(t)$ satisfy (2.1), where 
$C_{2} \in C^{1}\left(\left[t_{+}, \infty\right),(0, \infty)\right)$. Let the conditions 1), 2) of Theorem 3.1 or 1), 2) of Theorem 3.2 be fulfilled, and the following conditions be also fulfilled:

3) there exists a region $\Omega \subset \mathbb{R}^{n}$ such that $0 \notin \Omega$ and the component $P_{1}(t) x(t)$ of each existing solution $x(t)$ with the initial point $\left(t_{0}, x_{0}\right) \in L_{t_{+}}$, where $P_{1}\left(t_{0}\right) x_{0} \in \Omega$, remains all the time in $\Omega$;

4) there exist a positive definite self-adjoint operator $H(t) \in L\left(\mathbb{R}^{n}\right)$ and functions $k \in C\left(\left[t_{+}, \infty\right), \mathbb{R}\right), \quad U \in C(0, \infty)$ such that $H \in C^{1}\left(\left[t_{+}, \infty\right), L\left(\mathbb{R}^{n}\right)\right)$, $\int_{t_{+}}^{+\infty} k(t) d t=\infty, \quad \int_{c}^{+\infty} \frac{d v}{U(v)}<\infty \quad(c=$ const $>0) \quad$ and $\quad$ the inequality $J\left(t, P_{1}(t) x\right) \geq k(t) U\left(\left(H(t) P_{1}(t) x, P_{1}(t) x\right)\right)$, where $J\left(t, P_{1}(t) x\right)$ is of the form (3.3), holds for all $(t, x) \in L_{t_{+}}$such that $P_{1}(t) x \in \Omega$.

Then for each initial point $\left(t_{0}, x_{0}\right) \in L_{t_{+}}$such that $P_{1}\left(t_{0}\right) x_{0} \in \Omega$ there exists a unique solution of the initial value problem (1.1), (1.2) and this solution is Lagrange unstable.

Proof. It is proved in the same way as in Theorem 3.1 (see p. 252) that there exists the unique solution $z=\zeta(t)$ of the equation (3.12) on $\left[t_{0}, \omega\right)$ which satisfies the initial condition $\zeta\left(t_{0}\right)=P_{1}\left(t_{0}\right) x_{0}$, where $\left[t_{0}, \omega\right)$ is the maximal interval of the existence of the solution. Further, as in the proof of Theorem 3.1, we obtain that there exists the unique solution $x(t)=\zeta(t)+\gamma(t)$ of $(1.1)$ on $\left[t_{0}, \omega\right)$ which satisfies the initial condition (1.2). Recall that $z=\zeta(t)$ and $u=\gamma(t)$ (where $\gamma(t)=\eta(t, \zeta(t)))$ are the unique solution of the system $(3.6),(3.7)$ on $\left[t_{0}, \omega\right)$ which satisfies the initial conditions $\zeta\left(t_{0}\right)=P_{1}\left(t_{0}\right) x_{0}, \gamma\left(t_{0}\right)=P_{2}\left(t_{0}\right) x_{0}\left(\left(t_{0}, x_{0}\right) \in L_{t_{+}}\right.$, $\left.\zeta(t) \in X_{1}(t), \gamma(t) \in X_{2}(t)\right)$.

We prove that the solution $x(t)$ is Lagrange unstable.

By the condition of the theorem, there exists a domain $\Omega \subset \mathbb{R}^{n}$ such that $0 \notin \Omega$ and the component $P_{1}(t) x(t)$ of each existing solution $x(t)$ with the initial point $\left(t_{0}, x_{0}\right) \in L_{t_{+}}$, where $P_{1}\left(t_{0}\right) x_{0} \in \Omega$, remains all the time in $\Omega$. Since $\zeta(t)=P_{1}(t) x(t)$, then each solution $\zeta(t)$ of the equation (3.12) starting in the region $\Omega$ remains all the time in it. Introduce the positive definite scalar function (3.13): $V(t, z)=(H(t) z, z)$, where $H(t)$ is the operator satisfying the condition 4). The derivative of $V$ along the trajectories of the system (3.12) has the form (3.14). By the condition 4), the inequality

$$
\begin{array}{r}
V_{(3.12)}^{\prime}(t, \zeta(t))=\left(H^{\prime}(t) \zeta(t), \zeta(t)\right)+2(H(t) \zeta(t), \widetilde{\Pi}(t, \zeta(t)))=2 J\left(t, P_{1}(t) x(t)\right) \geq \\
\geq 2 k(t) U((H(t) \zeta(t), \zeta(t)))=2 k(t) U(V(t, \zeta(t))) .
\end{array}
$$

holds for all $t \geq t_{0}, \zeta(t) \in \Omega$. Hence, for $t \geq t_{0}$ the function $v(t)=V(t, \zeta(t))$ is a positive solution of the differential inequality

$$
v^{\prime} \geq 2 k(t) U(v) \text {. }
$$

It follows from the properties of the functions $k(t), U(v)$ that the inequality (3.24) $\left(t \geq t_{+}\right)$has no positive solution defined in the future [16]. Consequently, by Theorem [16, Ch. 4, Theorem XIV] the solution $\zeta(t)$ is Lagrange unstable, i.e., the maximal interval of existence $\left[t_{0}, \omega\right)$ is a finite interval and $\lim _{t \rightarrow \omega-0}\|\zeta(t)\|=\infty$. Hence, the solution $x(t)=\zeta(t)+\gamma(t)$ of (1.1), (1.2) is also Lagrange unstable (has the finite escape time $\left[t_{0}, \omega\right)$ ). 


\subsection{Dissipativity (ultimate boundedness).}

Ultimately bounded systems of (explicit) ordinary differential equations, which are also called dissipative systems or $D$-systems, were investigated, in particular, in $[16,29,20]$. Below are definitions similar to those given in $[16,29,20]$.

Definition 3.2. Solutions of the equation (1.1) are called ultimately bounded, if there exists a constant $K>0$ and for each solution $x(t)$ with an initial point $\left(t_{0}, x_{0}\right)$ there exists a number $\tau=\tau\left(t_{0}, x_{0}\right) \geq t_{0}$ such that $\|x(t)\|<K$ for all $t \in\left[t_{0}+\tau, \infty\right)$ (note that the constant $K$ is independent of the choice of a solution, i.e. the choice of $\left.t_{0}, x_{0}\right)$. The equation (1.1) is called ultimately bounded or dissipative, if for any consistent initial point $\left(t_{0}, x_{0}\right)$ there exists a global solution of the initial value problem (1.1), (1.2) and all solutions are ultimately bounded.

Definition 3.3. If in Definition 3.2 the number $\tau$ does not depend on the choice of $t_{0}$, i.e., $\tau=\tau\left(x_{0}\right)$, then the solutions of the equation (1.1) are called uniformly ultimately bounded and, accordingly, the equation (1.1) is called uniformly ultimately bounded or uniformly dissipative.

Analogous definitions hold for the equation (1.3).

Theorem 3.5. Let $f \in C\left(\left[t_{+}, \infty\right) \times \mathbb{R}^{n}, \mathbb{R}^{n}\right), \frac{\partial}{\partial x} f \in C\left(\left[t_{+}, \infty\right) \times \mathbb{R}^{n}, L\left(\mathbb{R}^{n}\right)\right)$, $A, B \in C^{1}\left(\left[t_{+}, \infty\right), L\left(\mathbb{R}^{n}\right)\right)$, the pencil $\lambda A(t)+B(t)$ satisfy (2.1), where $C_{2} \in C^{1}\left(\left[t_{+}, \infty\right),(0, \infty)\right)$, and the conditions 1), 2) of Theorem 3.1 or 1), 2) of Theorem 3.2 be fulfilled. Let the following conditions be also fulfilled:

3) there exist a positive definite self-adjoint operator $H(t) \in L\left(\mathbb{R}^{n}\right)$, a number $R>0$ and a function $U \in C(0, \infty)$ such that $H \in C^{1}\left(\left[t_{+}, \infty\right), L\left(\mathbb{R}^{n}\right)\right)$, sup $\|H(t)\|<\infty$ and for all $(t, x) \in L_{t_{+}},\left\|P_{1}(t) x\right\| \geq R$, one of the follow$t \in\left[t_{+}, \infty\right)$

ing inequalities (where $J\left(t, P_{1}(t) x\right)$ is of the form (3.3)) is fulfilled:

3. a) $J\left(t, P_{1}(t) x\right) \leq-U\left(\left(H(t) P_{1}(t) x, P_{1}(t) x\right)\right)$;

3.b) $J\left(t, P_{1}(t) x\right) \leq-U\left(\left\|P_{1}(t) x\right\|\right)$;

4) there exist a constant $c>0$ and a number $T>t_{+}$such that $\left\|G^{-1}(t) Q_{2}(t)\left[f\left(t, P_{1}(t) x+P_{2}(t) x\right)-A^{\prime}(t) P_{1}(t) x\right]\right\| \leq c\left\|P_{1}(t) x\right\|$ for all $(t, x) \in L_{T}\left(L_{T}\right.$ is $L_{t_{+}}$, where $t_{+}$is replaced by $\left.T\right)$.

Then the equation (1.1) is uniformly dissipative (uniformly ultimately bounded).

Proof. As in the proof of Theorem 3.1 or 3.2, we obtain that for each initial point $\left(t_{0}, x_{0}\right) \in L_{t_{+}}$there exists the unique global solution $x(t)=\zeta(t)+\gamma(t)$ of the initial value problem (1.1), (1.2), where $\gamma(t)=\eta(t, \zeta(t))\left(\zeta(t)=P_{1}(t) x(t), \gamma(t)=\right.$ $\left.P_{2}(t) x(t)\right)$. Indeed, since the inequality $V_{(3.12)}^{\prime}(t, \zeta(t)) \leq-2 U((H(t) \zeta(t), \zeta(t)))$ or $V_{(3.12)}^{\prime}(t, \zeta(t)) \leq-2 U(\|\zeta(t)\|)$ will be fulfilled instead of $(3.15)$, then the inequality $v^{\prime} \leq 0$, which also has no positive solution with finite escape time, will be fulfilled instead of (3.16). Moreover, by virtue of the condition 3), as in the proof of Yoshizawa's theorem [29, Theorem 10.4], we obtain that solutions of the ODE (3.12) are uniformly ultimately bounded, i.e., there exists a constant $N>0$ and for each solution $z=\zeta(t)$ satisfying the initial condition $\zeta\left(t_{0}\right)=P_{1}\left(t_{0}\right) x_{0}$ there exists a number $\tau_{1}=\tau_{1}\left(x_{0}\right) \geq t_{0}$ such that $\|\zeta(t)\|<N$ for all $t \geq t_{0}+\tau_{1}$. Although in Yoshizawa's theorem an inequality of the form 
$V_{(3.12)}^{\prime}(t, \zeta(t)) \leq-2 U(\|\zeta(t)\|)$ is used, but it is easy to verify that the statement remains true if $V_{(3.12)}^{\prime}(t, \zeta(t)) \leq-2 U((H(t) \zeta(t), \zeta(t)))$. Then, by the condition 4) and the equality (3.22), there exist a constant $c>0$ and a number $\tau_{2}=\tau_{2}\left(x_{0}\right)>t_{0}$ such that $\|\gamma(t)\|=\|\eta(t, \zeta(t))\| \leq c\|\zeta(t)\|<c N$ for all $t \geq \tau_{2}$. Thus, for each solution with the initial point $\left(t_{0}, x_{0}\right)$ there exists a number $\tau=\tau\left(x_{0}\right) \geq t_{0}$ such that $\|x(t)\| \leq\|\zeta(t)\|+\|\gamma(t)\|<(1+c) N$ for all $t \in\left[t_{0}+\tau, \infty\right)$, where the constant $(1+c) N>0$ is independent of $t_{0}, x_{0}$. Consequently, the equation (1.1) is uniformly dissipative (uniformly ultimately bounded).

\subsection{Global boundedness of solutions of the equation (1.3).}

Theorems similar to those proved above for the equation (1.1) are true for the nonautonomous degenerate DE (1.3). The theorems for the equation (1.3) are formulated as Theorems 3.1-3.5, where the following changes are made:

- in the condition 3) of Theorem 3.1, which is also contained in Theorems 3.2 and 3.3 , in the condition 4 ) of Theorem 3.4 and the condition 3 ) of Theorem 3.5, the function $J\left(t, P_{1}(t) x\right)$ (3.3) on the left-hand sides of the inequalities is replaced by

$$
\begin{aligned}
& K\left(t, P_{1}(t) x\right)=\frac{1}{2}\left(H^{\prime}(t) P_{1}(t) x, P_{1}(t) x\right)+ \\
& \quad+\left(H(t) P_{1}(t) x, G^{-1}(t)\left[-B(t) P_{1}(t) x+Q_{1}(t) f(t, x)\right]+P_{1}^{\prime}(t) x\right)
\end{aligned}
$$

- the manifold $L_{t_{+}}$is replaced by $\widehat{L}_{t_{+}}$and it is assumed that $f \in C^{1}\left(\left[t_{+}, \infty\right) \times \mathbb{R}^{n}, \mathbb{R}^{n}\right)$ everywhere;

- in Theorem 3.3, the condition a) takes the form $\sup _{(t, x) \in \widehat{L}_{t_{+}},\left\|P_{1}(t) x\right\| \leq M, M=\text { const }>0}$

and the requirement (3.20) is replaced by $\sup _{t \in\left[t_{+}, \infty\right),\left\|x_{p_{1}}(t)\right\| \leq M, M=\text { const }>0}\left\|G^{-1}(t) Q_{2}(t) f\left(t, x_{p_{1}}(t)+\tilde{x}_{p_{2}}\left(t_{*}\right)\right)\right\|<\infty ;$

- in Theorem 3.5, in the condition 4) the inequality $\left\|G^{-1}(t) Q_{2}(t)\left[f\left(t, P_{1}(t) x+P_{2}(t) x\right)-A^{\prime}(t) P_{1}(t) x\right]\right\| \leq c\left\|P_{1}(t) x\right\|$ is replaced by $\left\|G^{-1}(t) Q_{2}(t) f\left(t, P_{1}(t) x+P_{2}(t) x\right)\right\| \leq c\left\|P_{1}(t) x\right\|$ and the manifold $L_{T}$ is replaced by $\widehat{L}_{T}$.

The proofs of the theorems for the equation (1.3) are carried out in the same way as the proofs of the corresponding theorems for (1.1).

\section{The Lyapunov stability and asymptotic stability of solutions, and the complete stability of nonautonomous degenerate DEs}

Consider the equations (1.1), (1.3), where $f(t, x)$ is such that $f(t, 0) \equiv 0$. Such equations are called DAEs (degenerate DEs) of perturbed motion (by analogy with ODEs). They have the equilibrium state (equilibrium position, stationary solution) $x_{o}(t) \equiv 0$. Recall that the points $\left(t_{0}, x_{0}\right) \in L_{t_{+}}$and $\left(t_{0}, x_{0}\right) \in \widehat{L}_{t_{+}}$ are called consistent initial points for the initial value problems (1.1), (1.2) and 
(1.3), (1.2) respectively (see Remark 2.1). Obviously, for the considered equations, $(t, 0) \in L_{t_{+}}$and $(t, 0) \in \widehat{L}_{t_{+}}$for each $t \in\left[t_{+}, \infty\right)$.

The definitions of stability and asymptotic stability formulated below are similar to those given in $[3,15,19]$.

Let $f:\left[t_{+}, \infty\right) \times U_{R}(0) \rightarrow \mathbb{R}^{n}$, where $U_{R}(0)=\left\{x \in \mathbb{R}^{n} \mid\|x\|<R\right\}$.

Definition 4.1. The solution $x_{o}(t) \equiv 0$ of $(1.1)(f(t, 0) \equiv 0)$ is called Lyapunov stable (or simply stable) if for any $\varepsilon>0(\varepsilon<R), t_{0} \in\left[t_{+}, \infty\right)$ there exists a number $\delta=\delta\left(\varepsilon, t_{0}\right)>0(\delta \leq \varepsilon)$ such that for any consistent initial point $\left(t_{0}, x_{0}\right)$ satisfying the condition $\left\|x_{0}\right\|<\delta$ there exists a global solution $x(t)$ of the initial value problem (1.1), (1.2) and this solution satisfies the inequality $\|x(t)\|<\varepsilon$ for all $t \in\left[t_{0}, \infty\right)$. If, in addition, there exists $\widetilde{\delta}=\widetilde{\delta}\left(t_{0}\right)>0(\widetilde{\delta} \leq \delta)$ such that for each solution $x(t)$ with an initial point $\left(t_{0}, x_{0}\right)$ satisfying the condition $\left\|x_{0}\right\|<\widetilde{\delta}$ the requirement $\lim _{t \rightarrow \infty} x(t)=0$ is fulfilled, then the solution $x_{o}(t) \equiv 0$ is called asymptotically Lyapunov stable or simply asymptotically stable.

If in Definition $4.1 \delta$ is independent of $t_{0}$, i.e., $\delta=\delta(\varepsilon)$, then the solution is called uniformly Lyapunov stable or uniformly stable $\left(\right.$ on $\left[t_{+}, \infty\right)$ ).

Definition 4.2. The solution $x_{o}(t) \equiv 0$ of $(1.1)(f(t, 0) \equiv 0)$ is called Lyapunov unstable (or simply unstable) if for some $\varepsilon>0(\varepsilon<R), t_{0} \in\left[t_{+}, \infty\right)$ and any $\delta>0$ there exist a solution $x_{\delta}(t)$ of the initial value problem (1.1), (1.2) and a time moment $t_{1}>t_{0}$ such that $\left\|x_{0}\right\|<\delta$ and $\left\|x_{\delta}\left(t_{1}\right)\right\| \geq \varepsilon$.

The definition of asymptotic stability in the large or complete stability given below for a DAE is similar to the corresponding definition for an explicit ODE (see [13, Ch. I, Section 5] and [16, Ch. II, Section 13]).

Definition 4.3. Consider the equation (1.1), where $f(t, x)$ is defined on $\left[t_{+}, \infty\right) \times$ $\mathbb{R}^{n}$ and $f(t, 0) \equiv 0$. If the solution $x_{o}(t) \equiv 0$ of $(1.1)(f(t, 0) \equiv 0)$ is asymptotically stable and, moreover, for each point $\left(t_{0}, x_{0}\right) \in L_{t_{+}}$(i.e. for each consistent initial point) there exists a global solution $x(t)$ of the initial value problem (1.1), (1.2) and $\lim _{t \rightarrow \infty} x(t)=0$, then the solution $x_{o}(t) \equiv 0$ is called asymptotically stable in the large, and the equation (1.1) is called completely stable or asymptotically stable.

Similar definitions hold for the equation (1.3).

It is known that the Lyapunov stability of a nonzero (non-stationary) solution of a nonlinear ODE does not imply its boundedness on the whole domain of definition and, accordingly, the Lagrange stability. Also, in general the Lagrange stability of ODE solutions does not imply their Lyapunov stability. In the general case, the Lyapunov instability does not imply the Lagrange instability, but the converse statement is true.

Theorem 4.1. Let $f \in C\left(\left[t_{+}, \infty\right) \times U_{R}(0), \mathbb{R}^{n}\right), f(t, 0) \equiv 0, \frac{\partial}{\partial x} f \in C\left(\left[t_{+}, \infty\right) \times\right.$ $\left.U_{R}(0), L\left(\mathbb{R}^{n}\right)\right), A, B \in C^{1}\left(\left[t_{+}, \infty\right), L\left(\mathbb{R}^{n}\right)\right)$ and the pencil $\lambda A(t)+B(t)$ satisfy $(2.1)$, where $C_{2} \in C^{1}\left(\left[t_{+}, \infty\right),(0, \infty)\right)$. Let for each $t_{*} \in\left[t_{+}, \infty\right)$ and $x_{p_{1}}^{*}\left(t_{*}\right)=0$, $x_{p_{2}}^{*}\left(t_{*}\right)=0$ the operator $(3.2)$ has the inverse. Then the following statements are true:

(a) If there exist a positive definite self-adjoint operator $H(t) \in L\left(\mathbb{R}^{n}\right)$ and numbers $r_{1}, r_{2}>0, r_{1}+r_{2}<R$, such that $H \in C^{1}\left(\left[t_{+}, \infty\right), L\left(\mathbb{R}^{n}\right)\right)$ and for all 
$t \in\left[t_{+}, \infty\right), x \in B_{r_{1}, r_{2}}(0)=\left\{x \in \mathbb{R}^{n} \mid\left\|P_{i}(t) x\right\| \leq r_{i}, i=1,2\right\}$ the inequality
$J\left(t, P_{1}(t) x\right) \leq 0, \quad$ where $J\left(t, P_{1}(t) x\right)$ is of the form $(3.3)$,

holds, then the solution $x_{o}(t) \equiv 0$ of $(1.1)$ is Lyapunov stable.

(b) Let there exist a positive definite self-adjoint operator $H(t) \in L\left(\mathbb{R}^{n}\right)$, numbers $r_{1}, r_{2}>0, r_{1}+r_{2}<R$, and a function $W \in C\left(\left\{z \in \mathbb{R}^{n} \mid\|z\| \leq r_{1}\right\},[0, \infty)\right)$ such that $H \in C^{1}\left(\left[t_{+}, \infty\right), L\left(\mathbb{R}^{n}\right)\right), \sup _{t \in\left[t_{+}, \infty\right)}\|H(t)\|<\infty, W(z)>0$ for $z \neq 0$, $W(0)=0$, and for all $t \in\left[t_{+}, \infty\right), x \in B_{r_{1}, r_{2}}(0), P_{1}(t) x \neq 0$, the following inequality holds:

$J\left(t, P_{1}(t) x\right) \leq-W\left(P_{1}(t) x\right)$, where $J\left(t, P_{1}(t) x\right)$ is of the form (3.3);

let $G^{-1}(t) Q_{2}(t)\left[f\left(t, P_{1}(t) x+P_{2}(t) x\right)-A^{\prime}(t) P_{1}(t) x\right] \rightarrow 0$ uniformly in $t$

$$
\text { on }[T, \infty) \text { as } x \rightarrow 0 \text { for some } T>t_{+} \text {; }
$$

then the solution $x_{o}(t) \equiv 0$ of $(1.1)$ is asymptotically Lyapunov stable.

(c) The statement (a), where the function $J\left(t, P_{1}(t) x\right)$ in the inequality (4.1) is replaced by $K\left(t, P_{1}(t) x\right)$ (3.25), the equation (1.1) is replaced by (1.3), and it is additionally assumed that $f \in C^{1}\left(\left[t_{+}, \infty\right) \times U_{R}(0), \mathbb{R}^{n}\right)$, is true.

(d) The statement (b), where the inequality (4.2) and the requirement (4.3) are replaced respectively by

$K\left(t, P_{1}(t) x\right) \leq-W\left(P_{1}(t) x\right)$, where $K\left(t, P_{1}(t) x\right)$ is of the form (3.25),

and

$G^{-1}(t) Q_{2}(t) f\left(t, P_{1}(t) x+P_{2}(t) x\right) \rightarrow 0$ uniformly in $t$

$$
\text { on }[T, \infty) \text { as } x \rightarrow 0 \text { for some } T>t_{+},
$$

the equation (1.1) is replaced by (1.3), and it is additionally assumed that $f \in C^{1}\left(\left[t_{+}, \infty\right) \times U_{R}(0), \mathbb{R}^{n}\right)$, is true.

Proof. The proof of the statement (a).

As in the proof of Theorem 3.1, we consider the mappings (3.4), (3.5) and the system (3.6), (3.7), i.e., $z^{\prime}(t)=\Pi(t, z(t), u(t)), F(t, z(t), u(t))=0$. Obviously, $f(t, 0) \equiv 0$ if and only if $\Pi(t, 0,0) \equiv 0$ and $F(t, 0,0) \equiv 0$.

We will assume that $A(t)$ is not equal to zero or invertible for all $t$, because these cases correspond either to a purely algebraic equation or to a purely differential equation (ODE). In the case when $A(t)$ is invertible (for all $t$ ), the DAE (1.1) can be reduced to an ODE and then the classical Lyapunov theorems can be used. In the case when $A(t)$ is identically equal to zero, the implicit function theorems and the constructions similar to those given below are used. Thus, the theorem remains true for these special cases, but the proof of the theorem is of interest precisely to the DAE (degenerate DE). Therefore, in what follows, it is assumed that $X_{1}(t) \neq\{0\}$ and $X_{2}(t) \neq\{0\}$. Recall that the dimensions of the subspaces $X_{1}(t)$ and $X_{2}(t)$ are constant for all $t \in\left[t_{+}, \infty\right)$ (see Remark 3.1).

It is clear that there exist some regions $D^{z}, D^{u} \subset \mathbb{R}^{n}$ containing the origin for which the mappings $\Pi, F$ are defined, i.e., $P_{1}(t) D^{z}+P_{2}(t) D^{u} \subset U_{R}(0)$ and $\Pi(t, z, u):\left[t_{+}, \infty\right) \times D^{z} \times D^{u} \rightarrow \mathbb{R}^{n}, F(t, z, u):\left[t_{+}, \infty\right) \times D^{z} \times D^{u} \rightarrow \mathbb{R}^{n}$. The mappings $\Pi, F \in C\left(\left[t_{+}, \infty\right) \times D^{z} \times D^{u}, \mathbb{R}^{n}\right)$ are continuously differentiable in $z, u$ and 
the partial derivatives of $F(t, z, u)$ have the form (3.8), (3.9), where $\Phi_{t, P_{1}(t) z, P_{2}(t) u}$ is the operator (3.2). As in Theorem 3.1, denote $\tilde{\Phi}_{t, z, u}=\Phi_{t, P_{1}(t) z, P_{2}(t) u}$.

Obviously, Lemma 3.1 remains valid.

By the condition of the theorem, for each $t_{*} \in\left[t_{+}, \infty\right)$ the operator $\tilde{\Phi}_{t_{*}, 0,0}=$ $\Phi_{t_{*}, 0,0}$ is invertible. Therefore, for each point $(t, z, u)=\left(t_{*}, 0,0\right)$ the operator $\Psi_{t, z, u}=\frac{\partial}{\partial u} F(t, z, u)(3.11)$ is invertible. Let $t_{*} \in\left[t_{+}, \infty\right)$ be an arbitrary fixed element. Since $F\left(t_{*}, 0,0\right)=0$ and the conditions of the implicit function theorems are satisfied, then there exist neighborhoods $U_{\sigma_{1}}\left(t_{*}\right) \times U_{\delta_{1}}^{z}(0) \subset\left[t_{+}, \infty\right) \times D^{z}$ $\left(U_{\sigma_{1}}\left(t_{+}\right)=\left[t_{+}, t_{+}+\sigma_{1}\right)\right), U_{\gamma_{1}}^{u}(0) \subset D^{u}$ and a unique function $u=\mu(t, z) \in$ $C\left(U_{\sigma_{1}}\left(t_{*}\right) \times U_{\delta_{1}}^{z}(0), U_{\gamma_{1}}^{u}(0)\right)$ which is continuously differentiable in $z$ on $U_{\sigma_{1}}\left(t_{*}\right) \times$ $U_{\delta_{1}}^{z}(0)$, satisfies the equation (3.10) (i.e., $F(t, z, \mu(t, z))=0$ for $(t, z) \in U_{\sigma_{1}}\left(t_{*}\right) \times$ $\left.U_{\delta_{1}}^{z}(0)\right)$ and $\mu\left(t_{*}, 0\right)=0$. Since $u=\mu(t, z)$ satisfies $(3.10)$ for $(t, z) \in U_{\sigma_{1}}\left(t_{*}\right) \times$ $U_{\delta_{1}}^{z}(0)$, then $\mu(t, z) \in X_{2}(t)$ and $\left(t, P_{1}(t) z+\mu(t, z)\right) \in L_{t_{+}}$for each $(t, z) \in$ $U_{\sigma_{1}}\left(t_{*}\right) \times U_{\delta_{1}}^{z}(0)$. Thus, the following statement similar to Lemma 3.2 is proved: for each $t \in\left[t_{+}, \infty\right)$ and each $z$ from the sufficiently small neighborhood $U_{\delta_{1}}^{z}(0)$ there exists a unique $u$ from the sufficiently small neighborhood $U_{\gamma_{1}}^{u}(0)$, satisfying (3.10). Since the obtained implicit function $u=\mu(t, z)$ is continuous at the point $\left(t_{*}, 0\right)$, then for every $\varepsilon_{1}>0$ there are $\tilde{\sigma}_{1}=\tilde{\sigma}_{1}\left(\varepsilon_{1}, t_{*}\right)>0, \tilde{\delta}_{1}=\tilde{\delta}_{1}\left(\varepsilon_{1}, t_{*}\right)>0$ $\left(\tilde{\sigma}_{1} \leq \sigma_{1}, \tilde{\delta}_{1} \leq \delta_{1}\right)$ such that $\|\mu(t, z)\|<\varepsilon_{1}$ for $(t, z) \in U_{\tilde{\sigma}_{1}}\left(t_{*}\right) \times U_{\tilde{\delta}_{1}}^{z}(0)$ and therefore $\|u\|<\varepsilon_{1}$ for $u=\mu(t, z)$. Thus, the following lemma is proved.

Lemma 4.1. For any $\varepsilon_{u}>0, t \in\left[t_{+}, \infty\right)$ and any $z \in U_{\delta_{*}}^{z}(0)$, where $\delta_{*}>0$ is sufficiently small, there exists a unique $u \in U_{\varepsilon_{u}}^{u}(0)$ satisfying (3.10) and this $u$ belongs to $X_{2}(t)$ (i.e., $\|u\|<\varepsilon_{u}, F(t, z, u)=0$ and $\left.u=P_{2}(t) u\right)$.

Let $\varepsilon>0(\varepsilon<R)$ be an arbitrary number. We represent it as the sum $\varepsilon=\varepsilon_{z}+\varepsilon_{u}$ of numbers $\varepsilon_{z}>0, \varepsilon_{u}>0$ which will be indicated below.

Using the implicit function theorems and Lemma 4.1, we obtain the following statement. For any fixed $t_{*} \in\left[t_{0}, \infty\right)$ there exist an interval $U_{\sigma_{2}}\left(t_{*}\right) \subset\left[t_{+}, \infty\right)\left(\sigma_{2}=\sigma_{2}\left(\varepsilon_{u}, t_{*}\right), U_{\sigma_{2}}\left(t_{+}\right)=\left[t_{+}, t_{+}+\sigma_{2}\right)\right)$, a neighborhood $U_{\delta_{2}}^{z}(0)\left(\delta_{2}=\delta_{2}\left(\varepsilon_{u}, t_{*}\right) \leq \varepsilon_{z}\right)$ and a unique function $\nu_{t_{*}}(t, z) \in C\left(U_{\sigma_{2}}\left(t_{*}\right) \times U_{\delta_{2}}^{z}(0), U_{\varepsilon_{u}}^{u}(0)\right)$ which is a solution of the equation (3.10) with respect to $u$ (i.e., $F\left(t, z, \nu_{t_{*}}(t, z)\right)=0$ for $(t, z) \in U_{\sigma_{2}}\left(t_{*}\right) \times U_{\delta_{2}}^{z}(0)$ ), is continuously differentiable in $z$ and belongs to $X_{2}(t)$ for each $(t, z) \in U_{\sigma_{2}}\left(t_{*}\right) \times U_{\delta_{2}}^{z}(0)$, as well as satisfies the equality $\nu_{t_{*}}\left(t_{*}, 0\right)=0$. Introduce the function $u=\eta(t, z):\left[t_{+}, \infty\right) \times U_{\delta_{2}}^{z}(0) \rightarrow U_{\varepsilon_{u}}^{u}(0)$ and define by $\eta(t, z)=\nu_{t_{*}}(t, z)$ at the point $(t, z)=\left(t_{*}, z_{*}\right)$ for each point $\left(t_{*}, z_{*}\right) \in\left[t_{+}, \infty\right) \times U_{\delta_{2}}^{z}(0)$. Then the function $u=\eta(t, z)$ is continuous in $(t, z)$, continuously differentiable in $z$ and a unique solution of the equation (3.10), and $\eta(t, z) \in X_{2}(t)$ for each $(t, z) \in\left[t_{+}, \infty\right) \times U_{\delta_{2}}^{z}(0)$ as well as $\eta(t, 0) \equiv 0$.

Substitute the function $u=\eta(t, z)$ in (3.4) and denote $\widetilde{\Pi}(t, z)=\Pi(t, z, \eta(t, z))$, then the equation (3.6) takes the form (3.12), i.e., $z^{\prime}(t)=\widetilde{\Pi}(t, z(t))$. By the properties of $\eta$ and $\Pi$, the function $\widetilde{\Pi}$ is continuous in $(t, z)$ and continuously differentiable in $z$ on $\left[t_{+}, \infty\right) \times U_{\delta_{2}}^{z}(0)$, and $\widetilde{\Pi}(t, 0) \equiv 0$. Clearly, for each initial point $\left(t_{0}, z_{0}\right) \in\left[t_{+}, \infty\right) \times U_{\delta_{2}}^{z}(0)$ there exists a unique local solution of (3.12).

Take any initial value $t_{0} \in\left[t_{+}, \infty\right)$ and choose any consistent initial value $x_{0}$, i.e., $\left(t_{0}, x_{0}\right) \in L_{t_{+}}$or $F\left(t_{0}, P_{1}\left(t_{0}\right) x_{0}, P_{2}\left(t_{0}\right) x_{0}\right)=0$, satisfying 
the condition $\left\|x_{0}\right\|<\delta \leq \varepsilon$, where $\delta=\delta\left(\varepsilon, t_{0}\right)>0$ is chosen so that $\left\|P_{1}\left(t_{0}\right) x_{0}\right\|<\delta_{z} \leq \min \left\{\varepsilon_{z}, \delta_{2}\right\}, \delta_{z}$ is a sufficiently small number which will be determined below, and $\left\|P_{2}\left(t_{0}\right) x_{0}\right\|<\varepsilon_{u}$. Denote $z_{0}=P_{1}\left(t_{0}\right) x_{0}$ and $u_{0}=P_{2}\left(t_{0}\right) x_{0}$, then $\eta\left(t_{0}, z_{0}\right)=u_{0}\left(\right.$ since $\left.F\left(t_{0}, z_{0}, u_{0}\right)=0\right)$. For the chosen initial point $\left(t_{0}, z_{0}\right)$ there exists a unique local solution $z=\zeta(t)$ of (3.12) satisfying the initial condition $\zeta\left(t_{0}\right)=z_{0}$. Then the functions $z=\zeta(t), u=\eta(t, \zeta(t))$ are a unique local solution of the system (3.6), (3.7) satisfying the initial conditions $\zeta\left(t_{0}\right)=z_{0}$, $\eta\left(t_{0}, \zeta\left(t_{0}\right)\right)=u_{0}$, and by Lemma 3.1 the function $x(t)=\zeta(t)+\eta(t, \zeta(t))$ $\left(\zeta(t)=P_{1}(t) x(t) \in X_{1}(t), \eta(t, \zeta(t))=P_{2}(t) x(t) \in X_{2}(t)\right)$ is a unique local solution of (1.1) satisfying the initial condition (1.2), where $x_{0}=z_{0}+u_{0}$. Introduce the positive definite function (3.13), where $H(t)$ is the operator satisfying the conditions of the statement (a). Without loss of generality, we can assume that $\delta_{2} \leq r_{1}$ and $\varepsilon_{u} \leq r_{2}$, where the numbers $r_{1}, r_{2}$ are defined in the statement (a). By virtue of the condition (4.1), for any $t \in\left[t_{0}, \infty\right), z \in X_{1}(t)$ such that $\|z\|<\delta_{2}$ the derivative of $V$ along the trajectories of the system (3.12) (see (3.14)) satisfies the inequality

$$
V_{(3.12)}^{\prime}(t, z) \leq 0 \text {. }
$$

Recall that $\left\|z_{0}\right\|<\delta_{z} \leq \min \left\{\varepsilon_{z}, \delta_{2}\right\}$, where $z_{0}=P_{1}\left(t_{0}\right) x_{0}=\zeta\left(t_{0}\right)$. Further, as in the proof of Lyapunov's theorem on stability [17], we obtain that the number $\delta_{z}=\delta_{z}\left(\varepsilon_{z}, t_{0}\right)>0$ can be chosen such that the solution $z=\zeta(t)$ is global and $\|\zeta(t)\|<\varepsilon_{z}$ for all $t \in\left[t_{0}, \infty\right)$. This holds for any $\varepsilon_{z}>0$. Choose $\delta_{z}, \varepsilon_{z}$ and $\varepsilon_{u}$ such that $\|\zeta(t)\|<\varepsilon_{z}$ for $t \in\left[t_{0}, \infty\right),\|\eta(t, \zeta(t))\|<\varepsilon_{u}$ for $t \in\left[t_{0}, \infty\right)$ and $\|\zeta(t)\|<\varepsilon_{z}$, and $\varepsilon_{z}+\varepsilon_{u}=\varepsilon$. Then $\|x(t)\|=\|\zeta(t)+\eta(t, \zeta(t))\|<\varepsilon_{z}+\varepsilon_{u}=\varepsilon$ for all $t \in\left[t_{0}, \infty\right)$. Since $\varepsilon>0$ and $t_{0} \in\left[t_{+}, \infty\right)$ were chosen arbitrarily, the statement (a) is proved.

The proof of the statement (b).

The Lyapunov stability of the zero solution is proved in the same way as above. We show that the solution $x(t)=\zeta(t)+\eta(t, \zeta(t))$ with the initial point $\left(t_{0}, x_{0}\right)$ $\left(x_{0}=z_{0}+u_{0}\right)$, constructed in the proof of the statement $(\mathrm{a})$, satisfies the requirement $\lim _{t \rightarrow \infty} x(t)=0$ for $\left\|x_{0}\right\|<\delta$ and a sufficiently small $\delta=\delta\left(t_{0}\right)>0$. As above, $\delta$ is chosen so that $\left\|z_{0}\right\|=\left\|P_{1}\left(t_{0}\right) x_{0}\right\|<\delta_{z}$, where $\delta_{z}$ is sufficiently small number which will be defined below. It is clear that the mentioned $\delta$ and $\delta_{z}$ are different from those chosen in the proof of the statement (a), but for convenience we retain the previous notation.

Since, by the condition of the statement (b), $H_{1}=\sup _{t \in\left[t_{+}, \infty\right)}\|H(t)\|<\infty$ and the function $V(t, z)=(H(t) z, z)$ satisfies the inequality $|V(t, z)| \leq H_{1}\|z\|^{2}$ for any $t \in\left[t_{+}, \infty\right)$ and $z \in \mathbb{R}^{n}$, then $V(t, z)$ has an infinitely small upper limit (in $\mathbb{R}^{n}$ ) (see the definition in [13, p. 11, Def. 1.7]). Since, by virtue of the condition (4.2), the inequality $V_{(3.12)}^{\prime}(t, z) \leq-W(z)$, where the scalar function $W(z)$ is continuous and positive definite, is fulfilled instead of (4.6), then, as in the proof of Lyapunov?s theorem on asymptotic stability [17], we obtain that the number $\delta_{z}=\delta_{z}\left(t_{0}\right)>0$ can be chosen such that $\lim _{t \rightarrow \infty} \zeta(t)=0$. Then, taking into account (4.3), (3.22) and $\eta(t, 0) \equiv 0$, we obtain that $\lim _{t \rightarrow \infty} \eta(t, \zeta(t))=0$. Consequently, $\lim _{t \rightarrow \infty} x(t)=0$, and the statement (b) is proved.

The statements (c) and (d) can be proved similarly. 
Theorem 4.2. Let $f \in C\left(\left[t_{+}, \infty\right) \times \mathbb{R}^{n}, \mathbb{R}^{n}\right), \quad f(t, 0) \equiv 0, \frac{\partial}{\partial x} f \in C\left(\left[t_{+}, \infty\right) \times\right.$ $\left.\mathbb{R}^{n}, L\left(\mathbb{R}^{n}\right)\right), A, B \in C^{1}\left(\left[t_{+}, \infty\right), L\left(\mathbb{R}^{n}\right)\right)$ and the pencil $\lambda A(t)+B(t)$ satisfy $(2.1)$, where $C_{2} \in C^{1}\left(\left[t_{+}, \infty\right),(0, \infty)\right)$. Let the conditions 1), 2) of Theorem 3.1 or 1), 2) of Theorem 3.2 be fulfilled. Let there exist a positive definite selfadjoint operator $H(t) \in L\left(\mathbb{R}^{n}\right)$ and a function $W \in C\left(\mathbb{R}^{n},[0, \infty)\right)$ such that $H \in C^{1}\left(\left[t_{+}, \infty\right), L\left(\mathbb{R}^{n}\right)\right), \sup _{t \in\left[t_{+}, \infty\right)}\|H(t)\|<\infty, W(z)>0$ for $z \neq 0, W(0)=0$, and the inequality (4.2) holds for all $(t, x) \in L_{t_{+}}, P_{1}(t) x \neq 0$. Let (4.3) be fulfilled. Then the solution $x_{o}(t) \equiv 0$ of (1.1) is asymptotically stable in the large, i.e., the equation (1.1) is completely stable.

Proof. Since the conditions of the theorem include the conditions of the statement (b) of Theorem 4.1, then the zero solution of (1.1) is asymptotically stable.

As in the proof of Theorem 3.1 or 3.2 , where the inequality $v^{\prime} \leq 0$ holds instead of (3.16), we obtain that for each consistent initial point $\left(t_{0}, x_{0}\right)$ there exists a unique global solution $x(t)=\zeta(t)+\eta(t, \zeta(t))$ of the initial value problem (1.1), $(1.2)$, where $\zeta(t)=P_{1}(t) x(t), \eta(t, \zeta(t))=P_{2}(t) x(t)$. We prove that $\lim _{t \rightarrow \infty} x(t)=0$.

Since in the present theorem the equation (1.1), where $f(t, 0) \equiv 0$, is considered, then, as in Theorem 4.1, the function $u=\eta(t, z)$ is such that $\eta(t, 0) \equiv 0$. Note that for $t \geq t_{0}, \zeta(t) \neq 0$, the inequality $V_{(3.12)}^{\prime}(t, \zeta(t)) \leq-W(\zeta(t))$, where the function $W(z)$ is continuous and positive definite, holds (since (4.2) holds) and $V_{(3.12)}^{\prime}(t, 0) \equiv 0$. Since the operator $H(t)$ is positive definite and $\sup _{t \in\left[t_{+}, \infty\right)}\|H(t)\|<$ $\infty$, then the function $V(t, z)=(H(t) z, z)$ has an infinitely small upper limit in $\mathbb{R}^{n}$ (see the proof of the statement (b) of Theorem 4.1) and an infinitely large lower limit in $\mathbb{R}^{n}$ (see the definition in [13, p. 36, Def. 5.2]). Further, as in the proof of the Barbashin-Krasovsky theorem on asymptotic stability in the large [13, p. 36, Theorem 5.2], we obtain that $\lim _{t \rightarrow \infty} \zeta(t)=0$. Then, as in the proof of the statement (b) of Theorem 4.1, we obtain that $\lim _{t \rightarrow \infty} \eta(t, \zeta(t))=0$, and therefore $\lim _{t \rightarrow \infty} x(t)=0$.

Theorem 4.3. Let $f \in C^{1}\left(\left[t_{+}, \infty\right) \times \mathbb{R}^{n}, \mathbb{R}^{n}\right), \quad f(t, 0) \equiv 0, \quad A, B \quad \in$ $C^{1}\left(\left[t_{+}, \infty\right), L\left(\mathbb{R}^{n}\right)\right)$ and the pencil $\lambda A(t)+B(t)$ satisfy $(2.1)$, where $C_{2} \in$ $C^{1}\left(\left[t_{+}, \infty\right),(0, \infty)\right)$. Let the conditions 1), 2) of Theorem 3.1 or 1), 2) of Theorem 3.2, where $L_{t_{+}}$is replaced by $\widehat{L}_{t_{+}}$, be fulfilled. Let there exist a positive definite self-adjoint operator $H(t) \in L\left(\mathbb{R}^{n}\right)$ and a function $W \in C\left(\mathbb{R}^{n},[0, \infty)\right)$ such that $H \in C^{1}\left(\left[t_{+}, \infty\right), L\left(\mathbb{R}^{n}\right)\right), \sup _{t \in\left[t_{+}, \infty\right)}\|H(t)\|<\infty, W(z)>0$ for $z \neq 0, W(0)=0$,

and the inequality (4.4) holds for all $(t, x) \in \widehat{L}_{t_{+}}, P_{1}(t) x \neq 0$. Let (4.5) be fulfilled. Then the solution $x_{o}(t) \equiv 0$ of (1.3) is asymptotically stable in the large, i.e., the equation (1.3) is completely stable.

Proof. The proof is similar to the proof of Theorem 4.2.

\section{Lyapunov instability of nonautonomous degenerate DEs}

Remark 5.1. Since the Lagrange instability of a solution implies its Lyapunov instability, then the theorems on the Lagrange instability of the equations (1.1), 
(1.3) can also be considered as the theorems on the Lyapunov instability (see Definition 4.2).

Theorem 5.1. Let $f \in C\left(\left[t_{+}, \infty\right) \times U_{R}(0), \mathbb{R}^{n}\right)\left(U_{R}(0)=\left\{x \in \mathbb{R}^{n} \mid\|x\|<R\right\}\right)$, $f(t, 0) \equiv 0, \frac{\partial}{\partial x} f \in C\left(\left[t_{+}, \infty\right) \times U_{R}(0), L\left(\mathbb{R}^{n}\right)\right), A, B \in C^{1}\left(\left[t_{+}, \infty\right), L\left(\mathbb{R}^{n}\right)\right)$ and the pencil $\lambda A(t)+B(t)$ satisfy $(2.1)$, where $C_{2} \in C^{1}\left(\left[t_{+}, \infty\right),(0, \infty)\right)$. Let for each $t_{*} \in\left[t_{+}, \infty\right)$ the operator $(3.2)$, where $x_{p_{1}}^{*}\left(t_{*}\right)=0$ and $x_{p_{2}}^{*}\left(t_{*}\right)=0$, has the inverse. Then the following statements are true:

(a) If there exist a positive definite self-adjoint operator $H(t) \in L\left(\mathbb{R}^{n}\right)$, numbers $r_{1}, r_{2}>0, r_{1}+r_{2}<R$, and a function $W \in C\left(\left\{z \in \mathbb{R}^{n} \mid\|z\| \leq r_{1}\right\},[0, \infty)\right)$ such that $H \in C^{1}\left(\left[t_{+}, \infty\right), L\left(\mathbb{R}^{n}\right)\right)$, $\sup _{t \in\left[t_{+}, \infty\right)}\|H(t)\|<\infty, W(z)>0$ for $z \neq 0$, $W(0)=0$, and for all $t \in\left[t_{+}, \infty\right), x \in B_{r_{1}, r_{2}}(0), P_{1}(t) x \neq 0 \quad\left(B_{r_{1}, r_{2}}(0)=\right.$ $\left.\left\{x \in \mathbb{R}^{n} \mid\left\|P_{i}(t) x\right\| \leq r_{i}, i=1,2\right\}\right)$, the inequality

$J\left(t, P_{1}(t) x\right) \geq W\left(P_{1}(t) x\right)$, where $J\left(t, P_{1}(t) x\right)$ is of the form (3.3),

holds, then the solution $x_{o}(t) \equiv 0$ of (1.1) is Lyapunov unstable.

(b) The statement (a), where the function $J\left(t, P_{1}(t) x\right)$ in the inequality (5.1) is replaced by $K\left(t, P_{1}(t) x\right)$ (3.25), the equation (1.1) is replaced by (1.3), and it is additionally assumed that $f \in C^{1}\left(\left[t_{+}, \infty\right) \times U_{R}(0), \mathbb{R}^{n}\right)$, is true.

Proof. The proof of the statement (a).

Let $\varepsilon_{u}>0$ be an arbitrary number satisfying $\varepsilon_{u} \leq r_{2}$. As in the proof of the statement (a) of Theorem 4.1 (where $\varepsilon_{z}=r_{1}$ ) we construct the function $\eta(t, z) \in C\left(\left[t_{+}, \infty\right) \times U_{\delta_{2}}^{z}(0), U_{\varepsilon_{u}}^{u}(0)\right)$, where $0<\delta_{2} \leq r_{1}$, such that $u=\eta(t, z)$ is continuously differentiable in $z$, belongs to $X_{2}(t)$ for each $(t, z) \in\left[t_{+}, \infty\right) \times U_{\delta_{2}}^{z}(0)$, satisfies the identity $\eta(t, 0) \equiv 0$ and is a unique solution of the equation (3.10). Here $r_{1}, r_{2}$ are the numbers defined in the statement (a).

Substituting the obtained function $u=\eta(t, z)$ in (3.4) and denoting $\widetilde{\Pi}(t, z)=$ $\Pi(t, z, \eta(t, z))$, we get the equation (3.12). Introduce the positive definite function (3.13), where $H(t)$ is the operator defined in the condition (a). By virtue of the condition $\sup _{t \in\left[t_{+}, \infty\right)}\|H(t)\|<\infty$, the function $V(t, z)$ has an infinitely small upper limit (in $\mathbb{R}^{n}$ ) (see the proof of the statement (b) of Theorem 4.1). By virtue of (5.1), for all $t \in\left[t_{+}, \infty\right)$ and all $z \in X_{1}(t)$ such that $0<\|z\|<\delta_{2}$ the derivative of $V$ along the trajectories of the system (3.12) satisfies the inequality $V_{(3.12)}^{\prime}(t, z) \geq W(z)$, where the function $W(z)$ is continuous and positive definite. Thus, $V(t, z)$ and $V_{(3.12)}^{\prime}(t, z)$ are positive for all $t \in\left[t_{+}, \infty\right)$ and all $z \in X_{1}(t)$ such that $0<\|z\|<\delta_{2}$.

As in the proof of the statement (a) of Theorem 4.1 we obtain that for any consistent initial point $\left(t_{0}, x_{0}\right)$ satisfying the condition $\left\|x_{0}\right\|<\Delta$, where $\Delta=\delta_{2}+$ $\varepsilon_{u}>0$ is chosen so that $\left\|P_{1}\left(t_{0}\right) x_{0}\right\|<\delta_{2}$ and $\left\|P_{2}\left(t_{0}\right) x_{0}\right\|<\varepsilon_{u}$, there exists a unique local solution $z=\zeta(t), u=\eta(t, \zeta(t))$ of the system (3.6), (3.7) satisfying the initial conditions $\zeta\left(t_{0}\right)=z_{0}=P_{1}\left(t_{0}\right) x_{0}, \eta\left(t_{0}, \zeta\left(t_{0}\right)\right)=u_{0}=P_{2}\left(t_{0}\right) x_{0}$. Then by Lemma 3.1 the function $x(t)=\zeta(t)+\eta(t, \zeta(t))$ is a unique local solution of (1.1) satisfying the initial condition (1.2), where $x_{0}=z_{0}+u_{0}$. Take an initial value $t_{0} \in\left[t_{+}, \infty\right)$, any consistent initial value $x_{0}\left(\left(t_{0}, x_{0}\right) \in L_{t_{+}}\right)$satisfying the condition $0<\left\|x_{0}\right\|<\delta(0<\delta<\Delta)$ and any (arbitrarily small) numbers $\delta_{z}>0$, 
$\delta_{u}>0$ such that $0<\left\|P_{1}\left(t_{0}\right) x_{0}\right\|<\delta_{z}<\delta_{2},\left\|P_{2}\left(t_{0}\right) x_{0}\right\|<\delta_{u}<\varepsilon_{u}$ and $\delta=\delta_{z}+\delta_{u}$. Taking into account the properties of the function $V(t, z)$ and its derivative along the trajectories of the system (3.12), as in the proof of Lyapunov?s theorem on instability [17], we obtain that for the solution $z=\zeta(t)$ of $(3.12)$ satisfying the initial condition $\zeta\left(t_{0}\right)=z_{0}=P_{1}\left(t_{0}\right) x_{0}$ and for some time moment $t_{1}>t_{0}$ the inequalities $0<\left\|z_{0}\right\|<\delta_{z}$ and $\left\|\zeta\left(t_{1}\right)\right\|>\delta_{2}$ hold. Consequently, for the corresponding solution $x(t)=\zeta(t)+\eta(t, \zeta(t))$ with the initial point $\left(t_{0}, x_{0}\right)$ the inequalities $\left\|x_{0}\right\|<\delta$ and $\left\|x\left(t_{1}\right)\right\|>\varepsilon=\delta_{2} /\left\|P_{1}\left(t_{1}\right)\right\|>0$ hold (since $\left\|\zeta\left(t_{1}\right)\right\|=$ $\left.\left\|P_{1}\left(t_{1}\right) x\left(t_{1}\right)\right\|\right)$. Since $\delta=\delta_{z}+\delta_{u}>0(\delta<\Delta)$ is arbitrary and $\varepsilon=\delta_{2} /\left\|P_{1}\left(t_{1}\right)\right\|>0$ is fixed, then the statement (a) is proved.

The statement (b) can be proved similarly.

\section{Application of the obtained theorems}

It is known that the dynamics of electrical circuits is modeled using systems of differential and algebraic equations, which in a vector form have the form of degenerate DEs or differential-algebraic equations. Generally, a degenerate DE describing the dynamics of an electrical circuit cannot be reduced to a purely differential equation, i.e., to an explicit ODE. In this section, we consider a simple electrical circuit with nonlinear and time-varying elements in order to demonstrate the application of the obtained theorems (on global solvability) and the verification of their conditions as well as to verify the adequacy of obtained restrictions (this is easily done by reducing the degenerate DE describing the circuit model to an explicit ODE). Namely, consider the electrical circuit with a time-varying inductance $L(t)$, time-varying linear resistances $r(t), r_{L}(t)$ and nonlinear resistances $\varphi_{L}\left(I_{L}\right), \varphi\left(I_{\varphi}\right)$, whose dynamics is described by the system of equations

$$
\begin{array}{r}
\frac{d}{d t}\left[L(t) x_{1}(t)\right]+r_{L}(t) x_{1}(t)-x_{2}(t)=-\varphi_{L}\left(x_{1}(t)\right), \\
x_{1}(t)+x_{3}(t)=I(t), \\
x_{2}(t)-r(t) x_{3}(t)=U(t)+\varphi\left(x_{3}(t)\right),
\end{array}
$$

where $I(t)$ is a given (input) current, $U(t)$ is a given (input) voltage, $x_{1}(t)=I_{L}(t)$ and $x_{3}(t)=I_{\varphi}(t)$ are unknown currents, and $x_{2}(t)=U_{L}(t)$ is an unknown voltage. The remaining currents and voltages in the circuit are uniquely expressed in terms of $I(t), I_{L}(t), I_{\varphi}(t), U(t)$ and $U_{L}(t)$.

In what follows, for brevity we omit the dependence on $t$ in the notation of the variables $x_{i}(t), i=1,2,3$.

The vector form of the system (6.1)-(6.3) is the nonautonomous degenerate DE (1.1), where $x=\left(x_{1}, x_{2}, x_{3}\right)^{T}=\left(I_{L}, U_{L}, I_{\varphi}\right)^{T} \in \mathbb{R}^{3}$,

$$
A(t)=\left(\begin{array}{ccc}
L(t) & 0 & 0 \\
0 & 0 & 0 \\
0 & 0 & 0
\end{array}\right), B(t)=\left(\begin{array}{ccc}
r_{L}(t) & -1 & 0 \\
1 & 0 & 1 \\
0 & 1 & -r(t)
\end{array}\right), f(t, x)=\left(\begin{array}{c}
-\varphi_{L}\left(x_{1}\right) \\
I(t) \\
U(t)+\varphi\left(x_{3}\right)
\end{array}\right) .
$$

It is assumed that $A, B \in C^{1}\left(\left[t_{+}, \infty\right), L\left(\mathbb{R}^{3}\right)\right), f \in C\left(\left[t_{+}, \infty\right) \times \mathbb{R}^{3}, \mathbb{R}^{3}\right)$ and $\frac{\partial}{\partial x} f \in C\left(\left[t_{+}, \infty\right) \times \mathbb{R}^{3}, L\left(\mathbb{R}^{3}\right)\right)$, i.e., $\quad L, r, r_{L} \in C^{1}\left(\left[t_{+}, \infty\right), \mathbb{R}\right)$, $I, U \in C\left(\left[t_{+}, \infty\right), \mathbb{R}\right)$ and $\varphi, \varphi_{L} \in C^{1}(\mathbb{R})$. 
Let $L(t) \neq 0$ for all $t \in\left[t_{+}, \infty\right)$, and $\lambda L(t)+r_{L}(t)+r(t) \neq 0$ for sufficiently large $|\lambda|$ such that $|\lambda| \geq \frac{1}{|L(t)|}$ and all $t \in\left[t_{+}, \infty\right)$. Then there exists the resolvent of the pencil $\lambda A(t)+B(t)$ :

$$
R(\lambda, t)=\frac{1}{\lambda L(t)+r_{L}(t)+r(t)}\left(\begin{array}{ccc}
1 & r(t) & 1 \\
-r(t) & {\left[\lambda L(t)+r_{L}(t)\right] r(t)} & \lambda L(t)+r_{L}(t) \\
-1 & \lambda L(t)+r_{L}(t) & -1
\end{array}\right)
$$

and it satisfies the estimate $(2.1)$, where $C_{2}(t)=\frac{1}{|L(t)|} \in C^{1}\left(\left[t_{+}, \infty\right),(0, \infty)\right)$ and $L(t)$ is either positive or negative for all $t \in\left[t_{+}, \infty\right)$. It is natural to take $L(t)>0$ $\left(t \in\left[t_{+}, \infty\right)\right)$ from the point of view of physics.

The projection matrices $P_{i}(t), \quad Q_{i}(t)$ (see (2.2)) have the form $P_{1}(t)=\left(\begin{array}{ccc}1 & 0 & 0 \\ -r(t) & 0 & 0 \\ -1 & 0 & 0\end{array}\right), \quad P_{2}(t)=\left(\begin{array}{ccc}0 & 0 & 0 \\ r(t) & 1 & 0 \\ 1 & 0 & 1\end{array}\right), \quad Q_{1}(t)=\left(\begin{array}{ccc}1 & r(t) & 1 \\ 0 & 0 & 0 \\ 0 & 0 & 0\end{array}\right)$, $Q_{2}(t)=\left(\begin{array}{ccc}0 & -r(t) & -1 \\ 0 & 1 & 0 \\ 0 & 0 & 1\end{array}\right)$

The vector $x$ has the projections $x_{p_{1}}(t)=P_{1}(t) x=\left(x_{1},-r(t) x_{1},-x_{1}\right)^{T}$, $x_{p_{2}}(t)=P_{2}(t) x=\left(0, r(t) x_{1}+x_{2}, x_{1}+x_{3}\right)^{T}$. Denote $a=x_{1}, b(t)=r(t) x_{1}+x_{2}$, $c=x_{1}+x_{3}$, then $x_{p_{1}}(t)=a(1,-r(t),-1)^{T}, x_{p_{2}}(t)=(0, b(t), c)^{T}$.

The consistency condition $(t, x) \in L_{t_{+}}$is satisfied if $(t, x)$ satisfies the algebraic equations (6.2), (6.3). Using the notation introduced above, the system (6.2), (6.3) can be rewritten as

$$
\begin{array}{r}
c=I(t), \\
b(t)=U(t)+r(t) I(t)+\varphi(I(t)-a) .
\end{array}
$$

Obviously, for each $t \in\left[t_{+}, \infty\right)$ and each $x_{1} \in \mathbb{R}$ there exist unique $x_{2} \in \mathbb{R}$ and $x_{3} \in \mathbb{R}$ such that the equalities (6.2), (6.3) hold, or for each $t \in\left[t_{+}, \infty\right)$ and each $a \in \mathbb{R}$ there exist unique $c \in \mathbb{R}$ and $b(t) \in \mathbb{R}$ such that the equalities (6.5), (6.6) hold. Consequently, the condition 1) of Theorem 3.1 is satisfied. It is clear that the condition 1) of Theorem 3.2 is also satisfied.

Take any fixed $t_{*}, x_{p_{1}}^{*}\left(t_{*}\right)=x_{1}^{*}\left(1,-r\left(t_{*}\right),-1\right)^{T}=a_{*}\left(1,-r\left(t_{*}\right),-1\right)^{T}, x_{p_{2}}^{*}\left(t_{*}\right)=$ $\left(0, r\left(t_{*}\right) x_{1}^{*}+x_{2}^{*}, x_{1}^{*}+x_{3}^{*}\right)^{T}=\left(0, b\left(t_{*}\right), c_{*}\right)^{T}$ such that $\left(t_{*}, x_{p_{1}}^{*}\left(t_{*}\right)+x_{p_{2}}^{*}\left(t_{*}\right)\right) \in L_{t_{+}}$ (i.e., $\left(t_{*}, x_{p_{1}}^{*}\left(t_{*}\right)+x_{p_{2}}^{*}\left(t_{*}\right)\right)$ satisfies $(6.2)$, (6.3) or (6.5), (6.6)). Consider the operator $\widehat{\Phi}_{t_{*}, x_{p_{1}}^{*}\left(t_{*}\right), x_{p_{2}}^{*}\left(t_{*}\right)}=\left[\frac{\partial}{\partial x}\left[Q_{2}\left(t_{*}\right) f\left(t_{*}, x_{p_{1}}^{*}\left(t_{*}\right)+x_{p_{2}}^{*}\left(t_{*}\right)\right)\right]-B\left(t_{*}\right)\right] P_{2}\left(t_{*}\right): \mathbb{R}^{3} \rightarrow$ $Y_{2}\left(t_{*}\right)$ and the operator $\Phi_{t_{*}, x_{p_{1}}^{*}\left(t_{*}\right), x_{p_{2}}^{*}\left(t_{*}\right)}=\left.\widehat{\Phi}\right|_{X_{2}\left(t_{*}\right)}: X_{2}\left(t_{*}\right) \rightarrow Y_{2}\left(t_{*}\right)$ defined in (3.2). With respect to the standard basis in $\mathbb{R}^{3}$, the matrix

$$
\begin{gathered}
\widehat{\Phi}_{t_{*}, x_{p_{1}}^{*}\left(t_{*}\right), x_{p_{2}}^{*}\left(t_{*}\right)}=\left(\begin{array}{ccc}
r\left(t_{*}\right)-\varphi^{\prime}\left(x_{3}^{*}\right) & 1 & -\varphi^{\prime}\left(x_{3}^{*}\right) \\
-1 & 0 & -1 \\
\varphi^{\prime}\left(x_{3}^{*}\right) & -1 & r\left(t_{*}\right)+\varphi^{\prime}\left(x_{3}^{*}\right)
\end{array}\right)= \\
=\left(\begin{array}{ccc}
r\left(t_{*}\right)-\varphi^{\prime}\left(I\left(t_{*}\right)-a_{*}\right) & 1 & -\varphi^{\prime}\left(I\left(t_{*}\right)-a_{*}\right) \\
-1 & 0 & -1 \\
\varphi^{\prime}\left(I\left(t_{*}\right)-a_{*}\right) & -1 & r\left(t_{*}\right)+\varphi^{\prime}\left(I\left(t_{*}\right)-a_{*}\right)
\end{array}\right)=\widetilde{\Phi}_{t_{*}, a_{*}}
\end{gathered}
$$


correspond to the operator $\widehat{\Phi}_{t_{*}, x_{p_{1}}^{*}}\left(t_{*}\right), x_{p_{2}}^{*}\left(t_{*}\right)$. It is easy to verify that from $\widehat{\Phi}_{t_{*}, x_{p_{1}}^{*}\left(t_{*}\right), x_{p_{2}}^{*}\left(t_{*}\right)} x_{p_{2}}(t)=0$ it follows that $x_{p_{2}}(t)=0$ for any $t$, and consequently there exists an inverse operator $\Phi_{t_{*}, x_{p_{1}}^{*}\left(t_{*}\right), x_{p_{2}}^{*}\left(t_{*}\right)}^{-1} \in L\left(Y_{2}\left(t_{*}\right), X_{2}\left(t_{*}\right)\right)$. Thus, the condition 2) of Theorem 3.1 is fulfilled.

Take any fixed $t_{*} \in\left[t_{+}, \infty\right), x_{p_{1}}^{*}\left(t_{*}\right) \in X_{1}\left(t_{*}\right), x_{p_{2}}^{i}\left(t_{*}\right) \in X_{2}\left(t_{*}\right)$ such that $\left(t_{*}, x_{p_{1}}^{*}\left(t_{*}\right)+x_{p_{2}}^{i}\left(t_{*}\right)\right) \in L_{t_{+}}, i=1,2$, i.e., any fixed $t_{*}, a_{*}, c_{*}^{i}, b^{i}\left(t_{*}\right), i=1,2$, such that the equalities (6.5), (6.6) hold. Since it follows from (6.5) that $c_{*}^{1}=c_{*}^{2}=I\left(t_{*}\right)$, then $c=I\left(t_{*}\right)$ for any $c \in\left[c_{*}^{1}, c_{*}^{2}\right]$. Take the projectors $\Theta_{k}\left(t_{*}\right) \in L\left(\mathbb{R}^{3}, Y_{2}\left(t_{*}\right)\right)\left(k=1,2, \Theta_{i}\left(t_{*}\right) \Theta_{j}\left(t_{*}\right)=\delta_{i j} \Theta_{i}\left(t_{*}\right), \sum_{k=1}^{2} \Theta_{k}\left(t_{*}\right)=Q_{2}\left(t_{*}\right)\right)$ to which the matrices $\Theta_{1}\left(t_{*}\right)=\left(\begin{array}{ccc}0 & -r(t) & 0 \\ 0 & 1 & 0 \\ 0 & 0 & 0\end{array}\right), \Theta_{2}\left(t_{*}\right)=\left(\begin{array}{ccc}0 & 0 & -1 \\ 0 & 0 & 0 \\ 0 & 0 & 1\end{array}\right)$ correspond with respect to the standard basis in $\mathbb{R}^{3}$. Then the system of the projectors $\left\{\tilde{\Theta}_{k}\left(t_{*}\right)=\left.\Theta_{k}\left(t_{*}\right)\right|_{Y_{2}\left(t_{*}\right)}\right\}_{k=1}^{2}$ is an additive resolution of the identity $\left.Q_{2}\left(t_{*}\right)\right|_{Y_{2}\left(t_{*}\right)}$ in $Y_{2}\left(t_{*}\right)$. Consider the operator function $\widehat{\Phi}_{t_{*}, x_{p_{1}}^{*}\left(t_{*}\right)}: X_{2}\left(t_{*}\right) \rightarrow L\left(\mathbb{R}^{3}, Y_{2}\left(t_{*}\right)\right)$, $\widehat{\Phi}_{t_{*}, x_{p_{1}}^{*}\left(t_{*}\right)}\left(x_{p_{2}}\left(t_{*}\right)\right)=\left[\frac{\partial}{\partial x}\left[Q_{2}\left(t_{*}\right) f\left(t_{*}, x_{p_{1}}^{*}\left(t_{*}\right)+x_{p_{2}}\left(t_{*}\right)\right)\right]-B\left(t_{*}\right)\right] P_{2}\left(t_{*}\right)$, and the operator function $\Phi_{t_{*}, x_{p_{1}}^{*}\left(t_{*}\right)}=\left.\widehat{\Phi}_{t_{*}, x_{p_{1}}^{*}\left(t_{*}\right)}\right|_{X_{2}\left(t_{*}\right)}: X_{2}\left(t_{*}\right) \rightarrow L\left(X_{2}\left(t_{*}\right), Y_{2}\left(t_{*}\right)\right)$ defined in (3.17). With respect to the standard basis in $\mathbb{R}^{3}$, the ma$\operatorname{trix} \widehat{\Phi}_{t_{*}, x_{p_{1}}^{*}\left(t_{*}\right)}\left(x_{p_{2}}\left(t_{*}\right)\right)=\widetilde{\Phi}_{t_{*}, a_{*}}$, where $\widetilde{\Phi}_{t_{*}, a_{*}}$ is introduced in $(6.7)$, correspond to the operator function $\widehat{\Phi}_{t_{*}, x_{p_{1}}^{*}\left(t_{*}\right)}\left(x_{p_{2}}\left(t_{*}\right)\right)$. Consider the operator $\Lambda=\left.\widehat{\Lambda}\right|_{X_{2}\left(t_{*}\right)} \in L\left(X_{2}\left(t_{*}\right), Y_{2}\left(t_{*}\right)\right)$, where $\widehat{\Lambda}=\Theta_{1}\left(t_{*}\right) \widehat{\Phi}_{t_{*}, x_{p_{1}}^{*}\left(t_{*}\right)}\left(x_{p_{2}, 1}\left(t_{*}\right)\right)+$ $\Theta_{2}\left(t_{*}\right) \widehat{\Phi}_{t_{*}, x_{p_{1}}^{*}\left(t_{*}\right)}\left(x_{p_{2}, 2}\left(t_{*}\right)\right)=\Theta_{1}\left(t_{*}\right) \widetilde{\Phi}_{t_{*}, a_{*}}+\Theta_{2}\left(t_{*}\right) \widetilde{\Phi}_{t_{*}, a_{*}}=\widetilde{\Phi}_{t_{*}, a_{*}}$ for any $x_{p_{2}, k}\left(t_{*}\right) \in\left[x_{p_{2}}^{1}\left(t_{*}\right), x_{p_{2}}^{2}\left(t_{*}\right)\right], k=1,2$. Since the operator $\Lambda$ is invertible, then the condition 2) of Theorem 3.2 is also fulfilled.

$$
\text { Choose } H(t)=\left(\begin{array}{ccc}
L(t) & 0 & 0 \\
0 & L(t) & 0 \\
0 & 0 & L(t)
\end{array}\right), t \in\left[t_{+}, \infty\right) \text {. Let } L(t) \geq L_{0}=\text { const }>0
$$

for all $t \in\left[t_{+}, \infty\right)$. Then $H(t) \in L\left(\mathbb{R}^{3}\right)$ is a positive definite operator. Let there exist a number $R>0$ such that

$$
\left[\varphi_{L}\left(x_{1}\right)-\varphi\left(I(t)-x_{1}\right)-r(t) I(t)-U(t)\right] x_{1}+\left[L^{\prime}(t) / 2+r_{L}(t)+r(t)\right] x_{1}^{2} \geq 0
$$

for all $t \in\left[t_{+}, \infty\right),\left\|x_{p_{1}}(t)\right\|=\left|x_{1}\right|\left\|(1,-r(t),-1)^{T}\right\| \geq R$. Then the condition 3$)$ from Theorems 3.1, 3.2, where $k(t)=\left|r^{\prime}(t) / r(t)\right|$ and $U(v)=v$, is fulfilled.

Thus, by Theorem 3.1 as well as by Theorem 3.2, for each initial point $\left(t_{0}, x^{0}\right) \in\left[t_{+}, \infty\right) \times \mathbb{R}^{3}$, where $x^{0}=\left(x_{1}^{0}, x_{2}^{0}, x_{3}^{0}\right)^{T}$, which satisfies (6.2), (6.3), where $t=t_{0}, x_{i}=x_{i}^{0}, i=1,2,3$, there exists a unique global solution $x(t)$ of the equation (1.1) satisfying the initial condition $x\left(t_{0}\right)=x^{0}$ if $L, r, r_{L} \in C^{1}\left(\left[t_{+}, \infty\right), \mathbb{R}\right)$, $I, U \in C\left(\left[t_{+}, \infty\right), \mathbb{R}\right), \varphi, \varphi_{L} \in C^{1}(\mathbb{R})$ and the following requirements are fulfilled: $L(t) \geq L_{0}>0$ for all $t \in\left[t_{+}, \infty\right) ; \lambda L(t)+r_{L}(t)+r(t) \neq 0$ for sufficiently large $|\lambda|$ such that $|\lambda| \geq L_{0}^{-1}$ and all $t \in\left[t_{+}, \infty\right)$; there exists a number $R>0$ such that (6.8) holds for all $t \in\left[t_{+}, \infty\right),\left\|x_{p_{1}}(t)\right\|=\left|x_{1}\right|\left\|(1,-r(t),-1)^{T}\right\| \geq R$. 


\section{Acknowledgements}

The research is provided by grant support of the National Academy of Sciences of Ukraine (project "Qualitative, asymptotic and numerical analysis of various classes of differential equations and dynamical systems, their classification, and practical application", state registration number 0119U102376).

\section{References}

[1] O. H. Asadova, N. G. Mammadova and A. Kh. Abbasova, Investigation of the mixed problem for the system of partial differential equations, Advanced Mathematical Models \& Applications 2 (2017), No. 2, 139-143.

[2] Yu. E. Boyarintsev, Methods for solving continuous and discrete problems for singular systems of equations (Russian), Nauka, Novosibirsk, 1996.

[3] V. F. Chistyakov and A. A. Shcheglova, Selected chapters of the theory of algebraicdifferential systems (Russian), Nauka, Novosibirsk, 2003.

[4] L. Dai, Singular control systems (Lecture notes in control and information sciences), Springer, Heidelberg, 1989.

[5] Ju. L. Daleckii and M. G. Krein, Stability of solutions of differential equations in Banach space, AMS, Providence, Rhode Island, 1974.

[6] M. S. Filipkovska, Continuation of solutions of semilinear differential-algebraic equations and applications in nonlinear radiotechnics (Russian), Visn. Kharkiv. Nats. Univ. Mat. Model. Inform. Tekh. Avt. Syst. Upr. 19 (2012), No. 1015, 306-319.

[7] M. S. Filipkovska, Two combined methods for the global solution of implicit semilinear differential equations with the use of spectral projectors and Taylor expansions, International Journal of Computing Science and Mathematics (in press), DOI: 10.1504/IJCSM.2019.10025236.

[8] M. S. Filipkovska, Lagrange stability of semilinear differential-algebraic equations and application to nonlinear electrical circuits, Journal of Mathematical Physics, Analysis, Geometry 14 (2018), No. 2, 169-196.

[9] M. S. Filipkovskaya, Lagrange stability and instability of irregular semilinear differential-algebraic equations and applications, Ukrainian Mathematical Journal, 70 (2018), 947-979.

[10] T. S. Gadjiev and M. N. Kerimova, Coercive estimate for degenerate elliptic parabolic equations, Proceedings of the Institute of Mathematics and Mechanics 41 (2015), No. 6, 123-134.

[11] Yu. E. Gliklikh, On global in time solutions for differential-algebraic equations, Vestnik YuUrGU. Ser. Mat. Model. Progr. 7 (2014), No. 3, 33-39.

[12] T. Kato, Perturbation theory for linear operators, Springer, Berlin, 1966.

[13] N. N. Krasovsky, Some of problems of the theory of stability of motion (Russian), Fizmatgiz, Moscow, 1959.

[14] P. Kunkel and V. Mehrmann, Differential-algebraic equations: analysis and numerical solution, EMS, Zurich, 2006.

[15] R. Lamour, R. März and C. Tischendorf, Differential-algebraic equations: A projector based analysis, Springer, Heidelberg, 2013.

[16] J. La Salle and S. Lefschetz, Stability by Liapunov's direct method with applications, Academic Press, New York, 1961.

[17] A. M. Lyapunov, The general problem of the stability of motion (Russian), Academy of Science, Moscow, 1950. [English translation: A. M. Lyapunov, The general problem of the stability of motion, International Journal of Control 55 (1992), No. 3, $521-772]$ 
[18] L. S. Pontryagin, Ordinary differential equations, Addison-Wesley, U.S.A., 1962.

[19] R. Riaza, Differential-algebraic systems: Analytical aspects and circuit applications, World Scientific Publishing Co. Pte. Ltd., Hackensack, NJ, 2008.

[20] R. Reissig, G. Sansone and R. Conti, Qualitative theory of nonlinear differential equations (Russian), Nauka, Moscow, 1974.

[21] A. G. Rutkas and M. S. Filipkovska, Extension of solutions of one class of differentialalgebraic equations (Russian), Zh. Obchysl. Prykl. Mat. 1 (2013), 135-145.

[22] A. G. Rutkas and L. A. Vlasenko, Existence of solutions of degenerate nonlinear differential operator equations, Nonlinear Oscillations 4 (2001), No. 2, 252-263.

[23] L. Schwartz, Analyse Mathématique, II, Hermann, Paris, 1967.

[24] R. E. Showalter, Degenerate parabolic initial-boundary value problems, Journal of Differential Equations 31 (1979), No. 3, 296-312.

[25] V. Tuan and P. V. Viet, Stability of solutions of a quasilinear index-2 tractable DAE by the Lyapunov second method, Ukrainian Mathematical Journal 56 (2004), No. 10, 1574-1593.

[26] L. A. Vlasenko, Evolution models with implicit and degenerate differential equations, Sistemnye Tekhnologii, Dniepropetrovsk, 2006.

[27] L. A. Vlasenko, A. D. Myshkis and A. G. Rutkas, On a class of differential equations of parabolic type with impulsive action, Differential Equations 44 (2008), 231-240.

[28] A. Yonchev, Linear perturbation bounds of the discrete-time LMI based bounded output energy control problem for descriptor systems, Advanced Mathematical Models 8 Applications 2 (2017), No. 1, 28-37.

[29] T. Yoshizawa, Stability theory by Liapunov's second method, Math. Soc. Jpn., Tokyo, 1966.

Maria S. Filipkovska (Filipkovskaya)

B. Verkin Institute for Low Temperature Physics and Engineering of the National Academy of Sciences of Ukraine, Kharkiv, 61103, Ukraine, V.N. Karazin Kharkiv National University, Kharkiv, 61022, Ukraine.

E-mail address: filipkovskaya@ilt.kharkov.ua

Received: March 18, 2020; Revised: July 11, 2020; Accepted: September 1, 2020 